\title{
An info-gap framework for robustness assessment of epistemic uncertainty models in hybrid structural reliability analysis
}

\author{
Antoine Ajenjo ${ }^{\mathrm{a}, \mathrm{b}, \mathrm{c}}$, Emmanuel Ardillon ${ }^{\mathrm{a}}$, Vincent Chabridon ${ }^{\mathrm{a}}$, Bertrand \\ Iooss $^{\mathrm{a}}$, Scott Cogan ${ }^{\mathrm{b}}$, Emeline Sadoulet-Reboul $^{\mathrm{b}}$ \\ ${ }^{a}$ EDF RED, 6 quai Watier, 78401 Chatou, France \\ ${ }^{b}$ Univ. Bourgogne Franche-Comté, CNRS/UFC/ENSMM/UTBM, \\ Department of Applied Mechanics, 24 rue de l'épitaphe, 25000 Besançon, France \\ ${ }^{c}$ Corresponding Author
}

\begin{abstract}
The main objective of this work is to study the impact of the choice of input uncertainty models on robustness evaluations for probabilities of failure. Aleatory and epistemic uncertainties are jointly propagated by considering hybrid models and applying random set theory. The notion of horizon of uncertainty found in the info-gap method, which is usually used to assess the robustness of a model to uncertainty, allows to compare the bounds on the probability of failure obtained from different epistemic uncertainty models at increasing levels of uncertainty. Info-gap robustness and opportuneness curves are obtained and compared for the interval model, the triangular and trapezoidal possibility distributions, the probabilistic uniform distribution and the parallelepiped convex model on two academic examples and one industrial use-case. A specific demand value, as introduced in the info-gap method, is used as a value of information metric to quantify the gain of information on the probability of failure between less informative uncertainty models and a more informative ones.
\end{abstract}

Keywords: hybrid structural reliability, epistemic uncertainty, robustness, info-gap, random sets

1. Introduction

Structural reliability [1] is of particular interest for risk-sensitive indus3 trial applications such as power generation [2] where system performance, 
and therefore safety, is subject to uncertainty. In this context, the safety is assessed by estimating reliability-oriented quantities of interest such as a low probability of failure or a high-order quantile on a specific output variable of interest. Two types of uncertainty are commonly distinguished, namely aleatory and epistemic [3]. Aleatory uncertainty is associated to natural randomness while epistemic uncertainty is understood as ignorance due to a lack of knowledge and is therefore potentially reducible. High-risk systems models are typical cases where epistemic uncertainty can be found as they often represent events that are rarely or never encountered. However, the potential impact of lack of knowledge must still be accounted for in order to make an informed decision on the safety of the system.

The notion of robustness has many interpretations and mathematical representations [4]. It is defined in this paper as the capacity of the system to fulfill a criterion despite differences between its predicted and operational behaviors which is a key point in engineering and more specifically in safety assessment. The info-gap framework [5] proposes a metric that quantifies the robustness of a possible decision to epistemic uncertainty by calculating its worst performance at increasing levels of uncertainty in order to privilege tolerance to unexpected situations over performance at a poor estimate of the system's environment [6]. Info-gap may be applied in a wide range of fields where decisions under severe uncertainty need to be made such as in structural design under seismic loads [7], climate policies [8] or water resource planning [9]. Its application to reliability quantities of interest such as probabilities of failure has been studied less. One example concering the reliability of penstocks can be found in [10] where epistemic uncertainty affects physical variables and input distribution parameters which can be seen as a parametric probability box problem.

While aleatory uncertainty is systematically treated using the probabilistic framework, many different, yet potentially related, representations are used to deal with epistemic uncertainty. If the info-gap framework chooses to use convex models of uncertainty [11], other representations such as interval model (which is a special case of convex models), Dempster-Shafer structures [12], possibility distributions [13] or probability boxes [14] are also common. Beer et al. [15] and Zio and Pedroni [16] propose reviews for such methods. In many applications, both types of uncertainty coexist. Therefore, the standard reliability analysis for which only aleatory uncertainty is modeled must be transformed to hybrid reliability analysis (HRA). A generalized framework is thus required to estimate hybrid reliability quantities of 
interest. Random set (RS) theory [17] provides such framework as it enables to represent and propagate combined uncertainty representations in order to estimate, for example, the bounds on the probability of failure.

A robustness analysis depends on how the epistemic uncertainty is modeled. Two different convex models may lead to different values of probabilities of failure which in turn leads to the following question: to what extent does the choice of the epistemic uncertainty representation affect a robustness analysis? In this paper, in the context of HRA, a methodology is proposed to assess, within the info-gap framework, the robustness of small probabilities of failure with respect to the choice of a specific representation of epistemic uncertainty in the inputs. To do so, several epistemic uncertainty models are considered using RS theory. This methodology enables to compare info-gap metrics - the so-called robustness and opportuneness curves and a value of information metric defined as the demand value - obtained from different uncertainty representations but also to highlight their implicit relationships.

The paper is organized as follows: Section 2 reviews the formulation of a HRA with the use of RS theory and ends with the main aspects of an info-gap analysis; Section 3 describes the framework that is used and how info-gap and RS theory are combined to compare uncertainty representations; Section 4 shows the results of the methodology applied to two academic examples and one industrial use-case that concerns the structural reliability of penstocks; finally Section 5 proposes some discussions about the presented work before concluding it in Section 6.

\section{Hybrid reliability analysis}

\subsection{List of common epistemic uncertainty representations}

The probabilistic framework is a very powerful and detailed way to model and propagate aleatory uncertainty. Appendix A briefly recalls how such framework may be used for standard reliability analysis and more specifically for estimating a probability of failure. Nevertheless, the exact knowledge of the joint probability density function (pdf) $f_{\mathbf{X}}$ requires the knowledge of the marginal pdf of each component $X_{i}$ and the dependence structure (i.e., the copula) between components which is often not known especially when only limited data is available. Epistemic uncertainty characterizes the lack of information as it is potentially reducible by gathering more knowledge.

As mentioned in the introduction, many types of epistemic models can be found in the literature depending on the nature of the uncertainty and the 
available information. The main properties of the uncertainty representations investigated in this paper, namely interval model, convex model, evidence theory, possibility distributions and probability box (p-box) theory, are given in Appendix B and may be further investigated by the reader with the corresponding references. In order to maintain a coherence in the rest of the paper, the variables that are modeled by such representations are described by the vector $\mathbf{Y}=\left(Y_{1}, Y_{2}, \ldots, Y_{n_{Y}}\right)^{\top}$.

Links between the different uncertainty models mentioned above. Fig. 1 summarizes the main links between the different uncertainty representations when considering structural reliability where each number has the following meaning:

1. Adds the information of dependency with a convex model and its coefficient of correlation;

2. Assigns weights to subsets of the interval with the mass distribution $\nu$;

3. Assigns a possibility distribution $\pi(\cdot)$ in the interval;

4. $Y^{L}=Y^{U}$;

5. Adds the information of the probability law;

6. $\bar{F}_{Y}=\underline{F}_{Y}$;

7. $\bar{F}_{Y}=\operatorname{Pl}(Y \leq y)$ and $\underline{F}_{Y}=\operatorname{Bel}(Y \leq y)$ (see [18] for more information);

8. Discretizes the support of the distribution into disjoint intervals of weight $\left.\nu\left(\left[y_{i}, y_{i+1}\right]\right)=\nu_{i}=\operatorname{Pr}(Y \in] y_{i}, y_{i+1}\right]$ ) (see [18] for more information);

9. $\bar{F}_{Y}=\mathrm{N}(Y \leq y)$ and $\underline{F}_{Y}=\Pi(Y \leq y)$ (see [18] for more information);

10. Discretizes the possibility distribution into nested intervals by equally discretizing the $\alpha$-axis and assign the weight $\nu_{i}=\alpha_{i}-\alpha_{i+1}$ to the corresponding interval $\left[y_{i}, \overline{y_{i}}\right]$ which is the $\alpha_{i}$-cut (see [18] for more information).

The figure can be read from top to bottom in terms of added information and a solid line represents the path from a less informative model to a more informative one. The dotted lines represent a path from a model to another without adding information. Note that a solid line could be added from the interval to the probability boxes but has been removed here to make the diagram easier to read. The comparison in terms of degree of information cannot be made in the proposed context between two models with no solid line joining them. For example, a convex model and the free p-box 


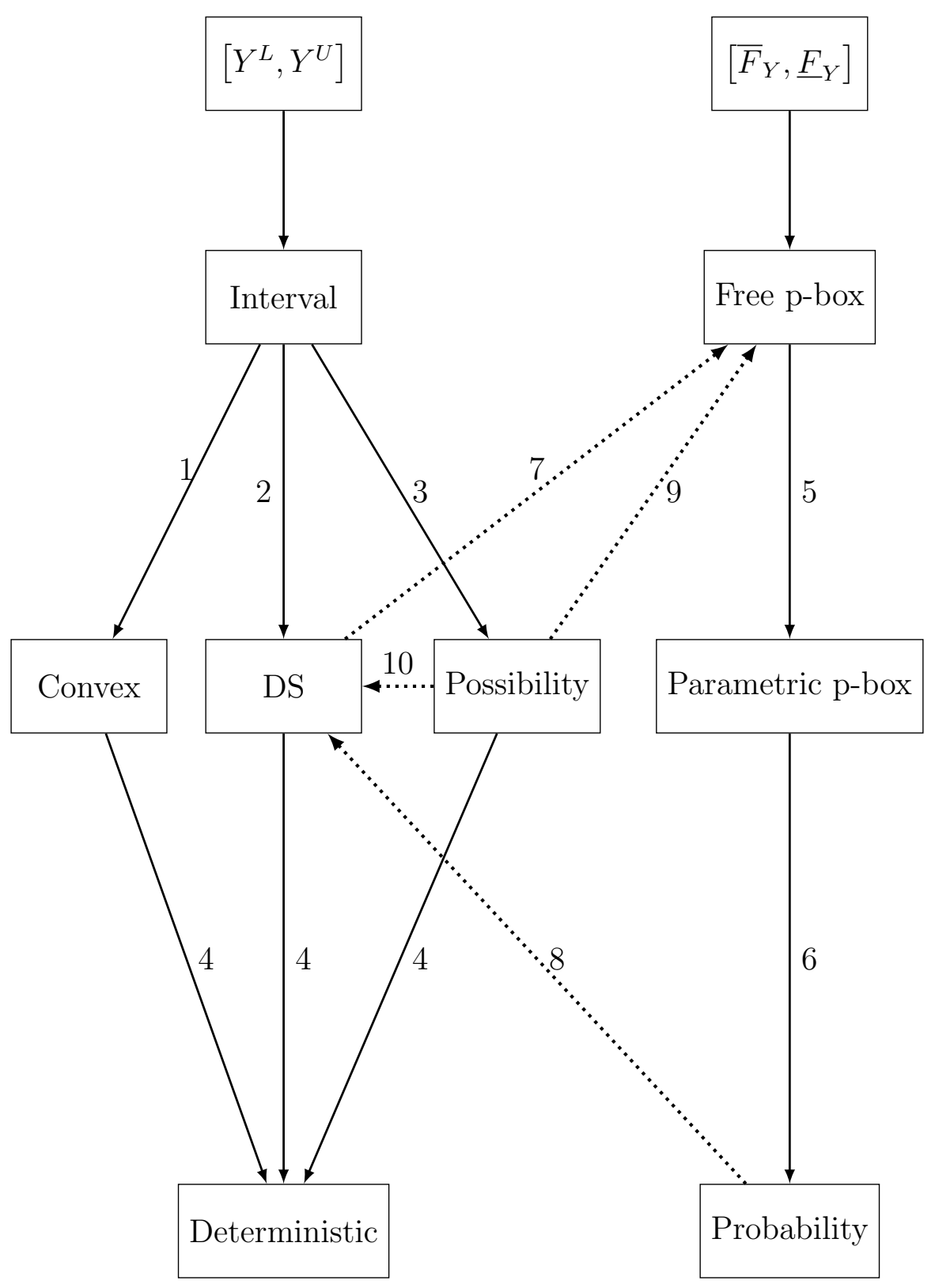

Figure 1: Diagram of various uncertainty representations.

113 representation are not directly comparable. Two groups of models may be distinguished in the diagram. On the left side, the uncertainty representa115 tions are interval-based. If no information is added to the bounds of the 
epistemic variables, the interval model and more generally the convex model may be used. DS structures and possibility distributions enable to divide the initial interval into more or less plausible smaller intervals based on expert knowledge. These representations reduce to a deterministic value with no epistemic uncertainty. On the right side, the uncertainty representations are probability-based. The path of information goes from the free to the parametric p-box representations and finally reduces to the purely probabilistic representation. Although these two groups of models seem to be built on different theoretical frameworks, the dotted lines show how they may be related in the context of reliability. Indeed, the following section explains how these models may be modeled in a common framework.

\subsection{Hybrid reliability analysis using random set theory}

This work falls in the scope of HRA, meaning that the input vector can be divided into two vectors, namely $\mathbf{X}$ and $\mathbf{Y}$ where $\mathbf{X}$ is a random vector with a fully determined pdf $f_{\mathbf{X}}$ and $\mathbf{Y}$ contains the input variables subject to epistemic uncertainty and described by one of the models mentioned previously. For a given realization of the random vector $\mathbf{X}$, the hybrid limit-state function $g(\mathbf{X}, \mathbf{Y})$ is also a random set. As such, it is not possible to compute a single probability of failure as in standard reliability analysis but only its bounds denoted by $\left[\underline{P_{\mathrm{f}}}, \overline{P_{\mathrm{f}}}\right]$ where:

$$
\begin{aligned}
& \underline{P_{\mathrm{f}}}=\operatorname{Pr}[\bar{g}(\mathbf{X}, \mathbf{Y})]=\operatorname{Pr}[\max g(\mathbf{X}, \mathbf{Y}) \leq 0] \\
& \overline{P_{\mathrm{f}}}=\operatorname{Pr}[\underline{g}(\mathbf{X}, \mathbf{Y})]=\operatorname{Pr}[\min g(\mathbf{X}, \mathbf{Y}) \leq 0] .
\end{aligned}
$$

In order to apply the existing probability of failure estimation methods to the hybrid problem, a framework that enables the propagation of random variables with a mixture of different epistemic models is needed. RS theory makes it possible $[19,20]$ as it generalizes probabilistic and epistemic models. A random set is very closely related to evidence theory and is defined by the function $\Gamma$ :

$$
\Gamma: \mid \begin{array}{lll}
\Omega & \longrightarrow \\
\boldsymbol{\alpha} & \longrightarrow & \Gamma(\boldsymbol{\alpha})
\end{array}
$$

where $A$ is the focal set and $\Gamma(\boldsymbol{\alpha})$ is a focal element. In other words, a random set is similar to a random variable whose realization is a set in $A$, not a scalar. The event $E$ is bounded by an upper probability and a lower 
probability that are quite similar to Eqs. (6.a) and (6.b):

$$
\begin{aligned}
& \frac{P_{\Gamma}}{\bar{P}}(E)=P_{\Omega}(\{\boldsymbol{\alpha} \in \Omega: \Gamma(\boldsymbol{\alpha}) \subseteq E, \Gamma(\boldsymbol{\alpha}) \neq \emptyset\}) \\
& \overline{P_{\Gamma}}(E)=P_{\Omega}(\{\boldsymbol{\alpha} \in \Omega: \Gamma(\boldsymbol{\alpha}) \cap E \neq \emptyset\})
\end{aligned}
$$

A RS can also be obtained from evidence theory by relating it to the p-box representation as shown in Fig. 1. The interval and convex models are special cases where the RS is actually a constant set as the function does not depend on $\boldsymbol{\alpha}$. The probability model is a special case where the random set is a singleton. A sample of the random set in higher dimension than one is obtained by sampling the vector $\boldsymbol{\alpha}$ from a copula $C$ and computing the Cartesian product $\times_{k=1}^{n_{\alpha}} \Gamma_{k}\left(\alpha_{k}\right)$ which is a $n_{\alpha}$-box with $n_{\alpha}=n_{X}+n_{Y}$ being the number of input variables. The limit-state functions in Eqs. (11.a) and (11.b) can be rewritten as follows:

$$
\begin{aligned}
& \bar{g}(\mathbf{X}, \mathbf{Y})=\bar{g}(\boldsymbol{\alpha})=\max _{\Gamma_{(X, Y)}(\boldsymbol{\alpha})} g(\boldsymbol{\alpha}) \\
& \underline{g}(\mathbf{X}, \mathbf{Y})=\underline{g}(\boldsymbol{\alpha})=\min _{\Gamma_{(X, Y)}(\boldsymbol{\alpha})} g(\boldsymbol{\alpha})
\end{aligned}
$$

which yields for the bounds on $P_{\mathrm{f}}$ :

$$
\begin{aligned}
& \overline{P_{\mathrm{f}}}=\int_{\Omega} \mathbb{1}_{\underline{g}(\boldsymbol{\alpha}) \leq 0} d C(\boldsymbol{\alpha}) \\
& \underline{P_{\mathrm{f}}}=\int_{\Omega} \mathbb{1}_{\bar{g}(\boldsymbol{\alpha}) \leq 0} d C(\boldsymbol{\alpha}) .
\end{aligned}
$$


The HRA problem thus reduces to two standard reliability analyses for which standard estimation methods may be used. It is important to notice, as pointed out in [21], that when considering only interval or parametric p-box models on $\mathbf{Y}$, the bounds obtained using the RS framework are larger than the ones obtained by applying a straightforward search for the maximum and minimum of the probability of failure in the interval domain (on the physical variables or the distributional parameters). For example, with the interval model, the following equations hold:

$$
\begin{aligned}
& \operatorname{Pr}\left[\underset{\max _{(X, Y)}(\boldsymbol{\alpha})}{ } g(\boldsymbol{\alpha}) \leq 0\right] \leq \min _{D_{Y}} \operatorname{Pr}[g(\mathbf{X}, \mathbf{Y}) \leq 0] \\
& \operatorname{Pr}\left[\min _{\Gamma_{(X, Y)}(\boldsymbol{\alpha})} g(\boldsymbol{\alpha}) \leq 0\right] \geq \max _{D_{Y}} \operatorname{Pr}[g(\mathbf{X}, \mathbf{Y}) \leq 0]
\end{aligned}
$$

When estimating the bounds of the probabilities of failure when considering the interval or distributional p-box models in the following application cases, the results obtained by applying $\mathrm{RS}$ theory will be compared with those obtained by performing a global optimization on the probability of failure using DIRECT algorithm [22].

\subsection{Robustness analysis}

Robustness analysis is of particular interest in engineering applications. A system is considered robust if small variations on an expected state of operation do not considerably deteriorate the expected performance. A robust solution may be preferable over a non-robust optimal solution [6]. The infogap framework aims at quantitatively measuring this notion of robustness in the context of decision making by introducing the following robustness function $h_{\mathrm{IG}}^{*}$ given by:

$$
h_{\mathrm{IG}}^{*}=\max _{h}\left\{\max _{\mathbf{u} \in U(h, \tilde{\mathbf{u}})} R(\mathbf{q}, \mathbf{u}) \leq r_{c}\right\}
$$

where $h_{\mathrm{IG}}^{*}$ is defined as the maximum amount of uncertainty that can be tolerated, i.e., for which the worst possible performance is still acceptable. Three components appear in the info-gap robustness function in Eq. (7):

- the performance function $R(\mathbf{q}, \mathbf{u})$ that evaluates the quantity of interest of a system of characteristic vector $\mathbf{q}$ at specific values of the uncertain vector $\mathbf{u}$; 
- the critical performance $r_{c}$ which is the value that the quantity of interest must not exceed (to be distinguished with the threshold $z_{\text {th }}$ introduced in Eq. (A.1)). Its value may be determined or not in an info-gap analysis;

- the uncertainty model $U(h, \tilde{\mathbf{u}})$ which is usually a non-probabilistic convex set, as introduced in Section 2.2, of horizon of uncertainty $h \in \mathbb{R}^{+}$containing the best estimation $\widetilde{\mathbf{u}}$ (nominal value of $\mathbf{u}$ ) of the uncertain vector $\mathbf{u}$. For $h=0, U(h, \widetilde{\mathbf{u}})$ reduces to $\widetilde{\mathbf{u}}$.

A key feature of the convex uncertainty models is that they are nested as the illustrative example depicted in Fig. 2:

$$
U\left(h_{1}, \tilde{\mathbf{u}}\right) \subseteq U\left(h_{2}, \tilde{\mathbf{u}}\right) \text { for } h_{1} \leq h_{2} .
$$

Therefore, the robustness function is monotonous with respect to the horizon of uncertainty and to the performance level.

Uncertainty can also be beneficial as the real performance of the system may be better than the expected one. To illustrate this point, the opportuneness function $\beta_{\mathrm{IG}}^{*}$ is defined as:

$$
\beta_{\mathrm{IG}}^{*}=\min _{h}\left\{\min _{\mathbf{u} \in U(h, \widetilde{\mathbf{u}})} R(\mathbf{q}, \mathbf{u}) \leq r_{w}\right\}
$$

where $r_{w}$ can be seen as a reward threshold. The idea with the IG framework is to compare the robustness values of different possible decisions $\mathbf{d}$ in order to retain the most robust one for a given critical performance value. The most robust decision may depend on the choice of the critical performance value as seen in Fig. 2 (right) where both curves cross each others. The decision $d_{2}$ is more robust before the curves intersect but the decision $d_{1}$ is more robust after. This crossing of robustness curves is called the preference reversal. Few hypotheses are required in an IG analysis as it can be conducted only with the choice of a non-probabilistic convex uncertainty model and the best guess of the uncertain vector $\mathbf{u}$, e.g., its nominal value. However, both hypotheses may have an influence on the robustness evaluation. The effect of the uncertainty model on the robustness curve can be seen as a value of information (VoI) analysis [23] where the aim is to quantify the gain in robustness when using a more informative uncertainty model than another 
one. An IG uncertainty model $U_{1}$ is more informative than $U_{2}$ if the following set inclusion is obtained:

$$
U_{1}(h, \tilde{\mathbf{u}}) \subset U_{2}(h, \tilde{\mathbf{u}}), \forall h \geq 0 .
$$

For a given critical performance $r_{c}, U_{1}$ will yield a higher robustness value. For a given horizon of uncertainty, the worst performance in $U_{1}$ will be better than the one in $U_{2}$. These comparisons are expressed respectively as the robustness premium $\Delta h^{*}$ and the demand value $\Delta r_{c}$.
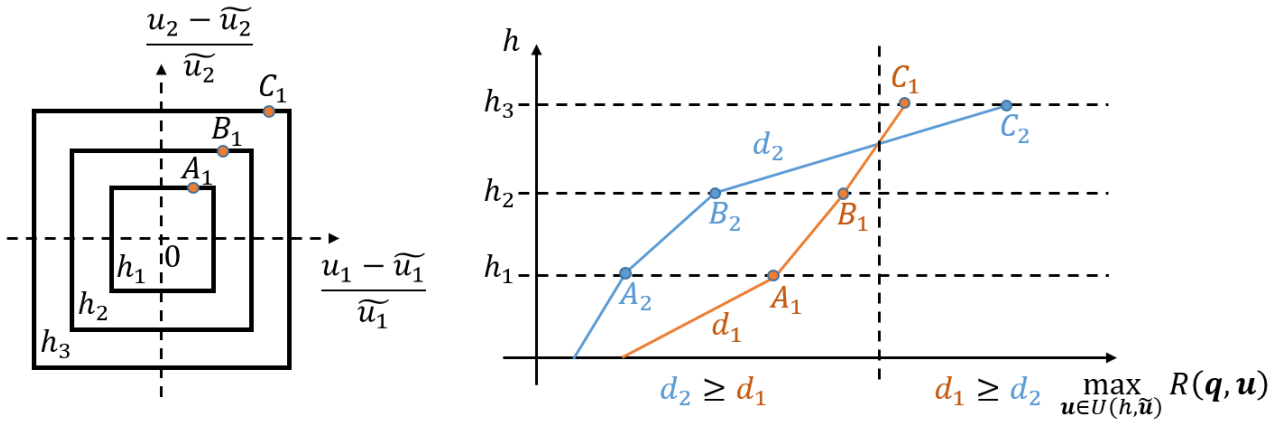

Figure 2: Nested convex sets (left) and associated robustness curves with preference reversal (right).

\section{A new framework for assessing robustness in hybrid reliability analysis}

\subsection{HRA framework}

The goal in this work is to analyse the effect of the choice of an epistemic uncertainty model on the robustness of a reliability quantity of interest. Here, one considers the bounds of the probability of failure obtained by HRA as the two quantities of interest. As mentioned in Section 2, the limit-state function $g(\mathbf{X}, \mathbf{Y})$ depends on both vectors $\mathbf{X}$ and $\mathbf{Y}$. The vector $\mathbf{X}$ contains the input variables $X_{i}$ that are modeled as random variables. The joint distribution $f_{\mathbf{X}}(\mathbf{x})$ is considered perfectly determined (no epistemic uncertainty). The vector $\mathbf{Y}$ contains the input variables $Y_{i}$ for which epistemic uncertainty does not allow a well defined deterministic or probabilistic modeling. As it was seen in Section 2, RS theory enables to model and propagate many different uncertainty models together (including probabilized inputs). In 
order to compare the effect of each epistemic uncertainty model, the bounds on the probability of failure are estimated and compared for a same epistemic representation of each input variable $Y_{i}$. The different epistemic models for which results are shown in this paper are:

- interval model;

- parallelepiped convex model;

- possibility triangular distribution;

- possibility trapezoidal distribution;

- DS structures;

- free and parametric p-boxes.

Probabilistic uniform distributions on $Y_{i}$ are also added to the comparison. In order to estimate the bounds on the probability of failure, Eqs. (15.a) and (15.b) need to be evaluated. The inner loop which corresponds to the search of the minimum and maximum of the limit-state function for one realization of the random set $\Gamma(\boldsymbol{\alpha})$ may be performed using an optimization algorithm. The outer loop corresponds to the estimation method of the probability of failure. As an inner optimization loop is involved, HRA usually requires more evaluations of the limit-state function than a standard reliability analysis. Moreover, the lower bound of the probability of failure to be estimated may be very small (e.g., such that $P_{\mathrm{f}}<10^{-5}$ ). Therefore, some estimation methods such as crude Monte Carlo sampling may not be practicable. In this paper, the outer loop is mainly performed with an Importance Sampling around the most probable failure point obtained with a FORM analysis [24]. The Subset Simulation algorithm [25] is also used in order to verify the results. However, note that several other advanced sampling methods could have been used here (e.g., directional sampling, line sampling) $[26]$.

\subsection{Comparison by means of info-gap robustness and opportuneness curves}

As seen in Section 2.3, the IG framework quantifies the notions of robustness and opportuneness to uncertainty by building nested convex sets around a nominal state which represents the analyst's best guess. An interesting feature is that it enables to compare different possible decisions 
in view of choosing the one that maximizes the robustness given a critical performance. IG analysis can also be used to assess the VoI induced by a more informative input model. Indeed, the different decisions can be directly linked to the choice of different uncertainty models $U_{i}(h, \widetilde{\mathbf{u}})$ that each has its own degree of information. Therefore, it is possible to compare robustness and opportuneness curves of different uncertainty models for $\mathbf{Y}$ by considering the random set function $\Gamma_{i}$ as the info-gap uncertainty model as follows:

$$
U_{i}(h, \widetilde{\mathbf{Y}})=\Gamma_{i}(\alpha, h)
$$

with:

$$
\Gamma_{i}: \mid \begin{aligned}
{[0,1]^{n_{Y}} \times \mathbb{R}^{+} } & \longrightarrow \operatorname{Supp}_{\mathbf{Y}}(h, \tilde{\mathbf{Y}}) \\
(\boldsymbol{\alpha}, h) & \longrightarrow \Gamma_{i}(\boldsymbol{\alpha}, h)
\end{aligned}
$$

where $[0,1]^{n_{Y}}$ is the unit hypercube and $\operatorname{Supp}_{\mathbf{Y}}$ is the support of $\mathbf{Y}$ that gets wider when the horizon of uncertainty $h$ increases:

$$
\operatorname{Supp}_{\mathbf{Y}}(h, \tilde{\mathbf{Y}})=\{\mathbf{Y}: \tilde{\mathbf{Y}}(1-h) \leq \mathbf{Y} \leq \tilde{\mathbf{Y}}(1+h)\}, h \geq 0 .
$$

The robustness function in Eq. (7) translates with the proposed methodology to:

$$
h_{\mathrm{IG}}^{*}=\max _{h}\left\{\bar{P}_{\mathrm{f}}\left(\Gamma_{i}\right) \leq P_{\mathrm{f}}^{\mathrm{cr}}\right\}
$$

where $P_{\mathrm{f}}^{\mathrm{cr}}$ is the critical probability of failure that may or may not be known. In practice, instead of searching for $h_{\mathrm{IG}}^{*}$, the robustness curve can be plotted by estimating $\bar{P}_{\mathrm{f}}$ for a certain number $n_{h}$ of horizons of uncertainty that belong to a chosen interval $h_{j} \in\left[0, h_{\max }\right], j=1, \cdots, n_{h}$. The same method can be applied to plot the opportuneness curve by estimating several times $\underline{P}_{\mathrm{f}}$. Note that in Eq. (12) the random set function is only applied on $\mathbf{Y}$ for the sake of conciseness. In the application cases, the random set function also takes into account the random variable $\mathbf{X}$ as in Eqs. (14.a) and (14.b).

Whatever the type of uncertainty model that is used for $\mathbf{Y}$, for a given level of horizon of uncertainty $h$, the same support is used to compare bounds obtained from each model which enables a meaningful comparison. Moreover, the fact that bounds are calculated for increasing horizons of uncertainty and, therefore, growing supports, enables a comparison in terms of robustness (upper bound $\overline{P_{\mathrm{f}}}$ ) and opportuneness (lower bound $\underline{P}_{\mathrm{f}}$ ) functions. The larger the support, the more impact the choice of the uncertainty model has on the bounds of the probability of failure. The following quantity $R_{\overline{P_{\mathrm{f}}}}^{(i j)}$ is defined 
in this paper as the demand value between a less informative uncertainty model $U_{i}$ and a more informative uncertainty model $U_{j}$ and is used as the VoI metric:

$$
R_{\overline{P_{\mathrm{f}}}}^{(i j)}=1-\frac{\overline{P_{\mathrm{f}}}\left(\Gamma_{i}(\boldsymbol{\alpha}, h)\right)}{\overline{P_{\mathrm{f}}}\left(\Gamma_{j}(\boldsymbol{\alpha}, h)\right)}
$$

The value of this metric, which is negative as $\overline{P_{\mathrm{f}}}\left(\Gamma_{i}(h)\right) \geq \overline{P_{\mathrm{f}}}\left(\Gamma_{j}(h)\right)$, shows how the added information from model $U_{i}$ to model $U_{j}$ diminishes, in terms of percentage, the upper bound of the probability of failure. A similar metric could be defined with the lower bound to quantify how a more informative model reduces the best possible outcome. This last metric is not used in this paper since, in the context of a reliability analysis, the main concern is to understand how the worst possible outcome may be reduced with more information.

\subsection{Sensitivity to the gain of information}

The field of sensitivity analysis has a very large background which is not discussed in this paper as it is not the main topic [27]. Sensitivity analysis aims at identifying the variables that have a significant impact on the quantity of interest in order to simplify the numerical model or to help the analyst decide where to judiciously allocate resources [28]. Many metrics exist depending on the analyst's objective. In this paper, a simple metric is defined in order to identify the epistemic variables where added information contributes the most to the global gain of information on the bounds of the probability of failure. The idea is then to compare the demand value $R_{\overline{P_{\mathrm{f}}}}$ obtained by considering a more informative uncertainty representation on one variable at a time and compare it with the value obtained when considering all variables at once. The following metric is defined:

$$
S_{Y_{k}}^{(i j)}=\frac{R_{\overline{P_{\mathrm{f}}}}^{(i j)(k)}}{R_{\overline{P_{\mathrm{f}}}}^{(i j)}} .
$$

where:

$$
R_{\overline{P_{\mathrm{f}}}}^{(i j)(k)}=1-\frac{\overline{P_{\mathrm{f}}}\left(\Gamma_{i}(\boldsymbol{\alpha}, h)\right)}{\overline{P_{\mathrm{f}}}\left(\Gamma_{j}^{(k)}(\boldsymbol{\alpha}, h)\right)}
$$

with $\Gamma_{j}^{(k)}=\left[\Gamma_{i}\left(\alpha_{1}, h\right), \cdots, \Gamma_{j}\left(\alpha_{k}, h\right), \cdots, \Gamma_{i}\left(\alpha_{n_{Y}}, h\right)\right]^{\top}$. This metric gives the contribution of information gained from an uncertainty model to a more 
informative one brought by each variable. The metric depends on the horizon of uncertainty which means that a variable may be informative for some range of horizon of uncertainty and less informative for other values. This characteristic can contribute valuable knowledge to the decision-making process.

\subsection{Proposed methodology}

This section aims at summarizing the steps that are followed to apply the proposed methodology to three reliability problems. The different steps are presented in Fig. 3 where each box is detailed as follows:

1. Compute the limit-state function $g(\mathbf{X}, \mathbf{Y})$, build a comparison group $\mathbf{G}$ that contains different uncertainty models $M_{i}$ to be compared, define the values of the horizon of uncertainty $h_{j}$ for which the bounds on the probability of failure will be estimated, associate the joint cumulative distribution function $F_{\mathbf{X}}(\mathbf{x})$ to $\mathbf{X}$ and the nominal values $\widetilde{\mathbf{Y}}$ to $\mathbf{Y}$;

2. Get the random set function $\Gamma_{i}$ that corresponds to the uncertainty model $M_{i}$ as presented in Table 1;

3. Compute the support $\operatorname{Supp}_{\mathbf{Y}}\left(h_{j}, \tilde{\mathbf{Y}}\right)$ as defined in Eq. 13 which enables to compute the random set function $\Gamma_{i}\left(\boldsymbol{\alpha}, h_{j}\right)$ as defined in Eq. 12 ;

4. For each discretized value $h_{j}$, estimate the bounds on the probability of failure where each random variable $\alpha_{k}$ follows the standard uniform distribution and each corresponding realization is either the maximum value of the limit-state function in $\Gamma_{i}\left(\boldsymbol{\alpha}, h_{j}\right)$ (estimation of $P_{\mathrm{f}}$ ) or the minimum value (estimation of $\overline{P_{\mathrm{f}}}$ ) obtained with an optimization algorithm. The privileged method used to estimate the probabilities is FORM-IS but SS is also used as a verification method. More details on the optimization and probabilities estimation algorithms are given in Section 4.1;

5. Once the bounds on the probability of failure are obtained for each discretized horizon of uncertainty $h_{j}$ and for each uncertainty model $M_{i}$, the VoI metric $R_{\overline{P_{\mathrm{f}}}}$, as defined in Eq. 15 , is evaluated;

6. Show the robustness $\left(\overline{P_{\mathrm{f}}}\right)$ and opportuneness $\left(P_{\mathrm{f}}\right)$ curves obtained with each uncertainty model $M_{i}$ and show the surface plot $R_{\overline{P_{\mathrm{f}}}}(h)$ which is a function of $h$ and the two different uncertainty models that are compared in terms of information; 
Note that the sensitivity analysis is not present in Fig. 3. The only addition is the estimation of $\overline{P_{\mathrm{f}}}\left(\Gamma_{j}^{(k)}(\boldsymbol{\alpha}, h)\right)$ in Eq. 17 which requires to consider the more informative model $M_{j}$ on one variable $Y_{k}$ at a time and compute $S_{Y_{k}}^{(i j)}$ as defined in Eq. 16.

\section{Applications}

\subsection{Test cases and numerical tools used}

The methodology that is proposed throughout this paper is applied on two academic examples, or toy-cases, and one industrial case which is relevant to the French electricity supplier EDF. The two academic examples correspond to modified versions of the three-dimensional Rosenbrock function and a two-degree-of-freedom oscillator system. The main objective is to compare robustness and opportuneness curves obtained from various uncertainty models with increasing level of informativeness. Therefore, the following groups of comparison are created:

- $\mathbf{M}_{1}$ : interval RS, interval DIRECT, trapezoidal possibility distribution, triangular possibility distribution, probabilistic uniform distribution;

- $\mathbf{M}_{2}$ : interval RS, parallelepiped convex model;

- $\mathbf{M}_{3}$ : free p-box, parametric p-box.

The group $\mathbf{M}_{1}$ corresponds to added information represented by the solid lines 3 and 4 in Fig. 1 and the solid line that could have been plotted between the interval and probability boxes. When considering the interval model, a distinction is made between "interval RS" which means that the bounds on the probability of failure are estimated using RS theory (left side of the inequalities in Eq. (16)) and "interval DIRECT" which means that the bounds are estimated by using the global optimization algorithm DIRECT directly on the probability of failure (right side of the inequalities in Eq. (16)). The group $\mathbf{M}_{2}$ corresponds to added information represented by the solid line 1 . The group $\mathbf{M}_{3}$ corresponds to added information represented by the solid line 5 .

Robustness and opportuneness curves are also presented in order to verify numerically the links between possibility distributions, DS theory, p-boxes and probability distributions. The following groups of comparison are defined for that purpose: 


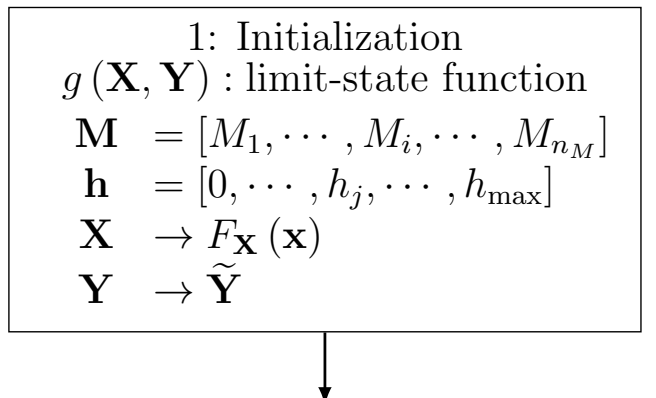

2: Association of the RS function with the uncertainty model $M_{i} \longleftrightarrow \Gamma_{i}$

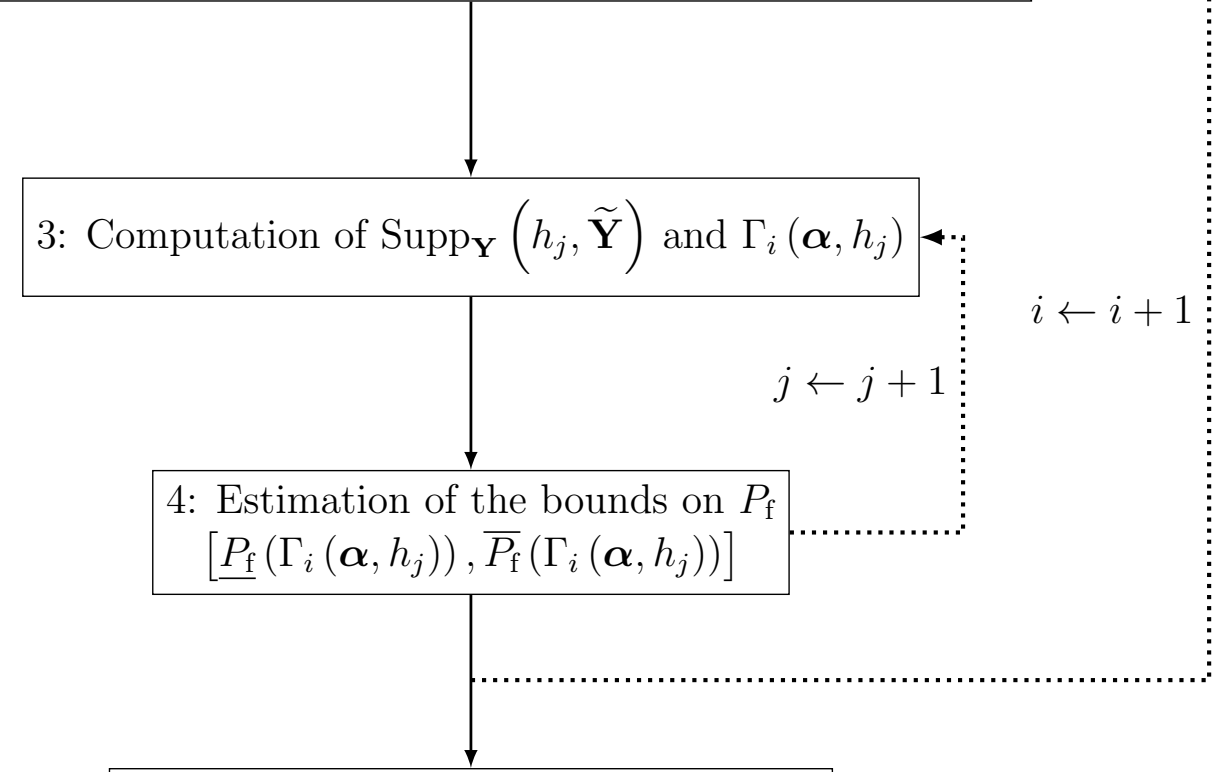

5: Computation of the VoI metric $R_{\frac{(i j)}{P_{\mathrm{f}}}}^{(i)}$

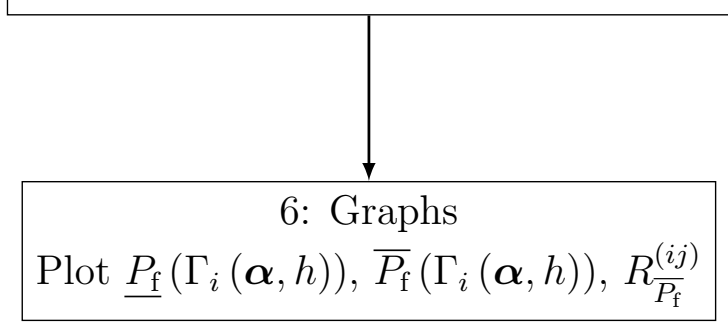

Figure 3: Workflow of the proposed methodology. 
- $\mathbf{M}_{4}$ : triangular possibility distribution, DS structures, free p-box;

- $\mathbf{M}_{5}$ : DS structures, probabilistic uniform distribution.

The group $\mathbf{M}_{4}$ corresponds to the dotted lines 7, 9 and 10 in Fig. 1. The group $\mathbf{M}_{5}$ corresponds to the dotted line 8 .

Parametric p-box results are also obtained using the DIRECT algorithm in the hyperrectangle resulting from each uncertain hyperparameter of the distribution law represented as a interval. For all the other models, the bounds are estimated using the RS framework. Moreover, hybrid limit-state functions are shown with the Rosenbrock function and the previously defined sensitivity and VoI metrics are computed. The methodology was numerically implemented with Python using mainly the Scipy package to solve the optimization problems (scipy.optimize module) arising from the hybrid limitstate functions, and the OpenTURNS software [29] to estimate probabilities of failure using mainly FORM-IS but also Subset Simulation for verification. When available, the robustness and opportuneness curves are given with their corresponding $95 \%$ confidence interval (dotted lines).

\subsection{Toy case 1: the Rosenbrock function}

The first toy case has the following limit-state function based on Rosenbrock function in three dimensions:

$$
g(\mathbf{X}, Y)=100\left(Y-X_{2}^{2}\right)^{2}+\left(X_{2}-1\right)^{2}+100\left(X_{2}-X_{1}^{2}\right)^{2}+\left(X_{1}-1\right)^{2}-3
$$

where $X_{1}$ and $X_{2}$ follow standard Gaussian distributions and $Y$ is the only epistemic variable with a nominal value of $Y^{C}=0.9$. Robustness and opportuneness curves are obtained by estimating the bounds $\left[\underline{P}_{\mathrm{f}}, \bar{P}_{\mathrm{f}}\right]$ for $n_{h}=15$ horizon levels for $h \in[0,0.19]$. As $Y$ has a single component, the convex model reduces to the interval model. The groups of uncertainty models on which results are provided are $\mathbf{M}_{1}, \mathbf{M}_{3}, \mathbf{M}_{4}$ and $\mathbf{M}_{5}$. The fact that the input dimension here is $n_{\alpha}=n_{X}+n_{Y}=3$ enables to draw the iso-lines of both limit-state surfaces $g(\boldsymbol{\alpha}, h)=0$ and $\bar{g}(\boldsymbol{\alpha}, h)=0$ in the $\boldsymbol{\alpha}$-space for different values of $h$ and different uncertainty models. Note that the $\boldsymbol{\alpha}$-space is the unit hypercube of dimension $n_{X}+n_{Y}$ and that $\alpha_{X_{i}}$ represents the quantile order of $X_{i}$. 


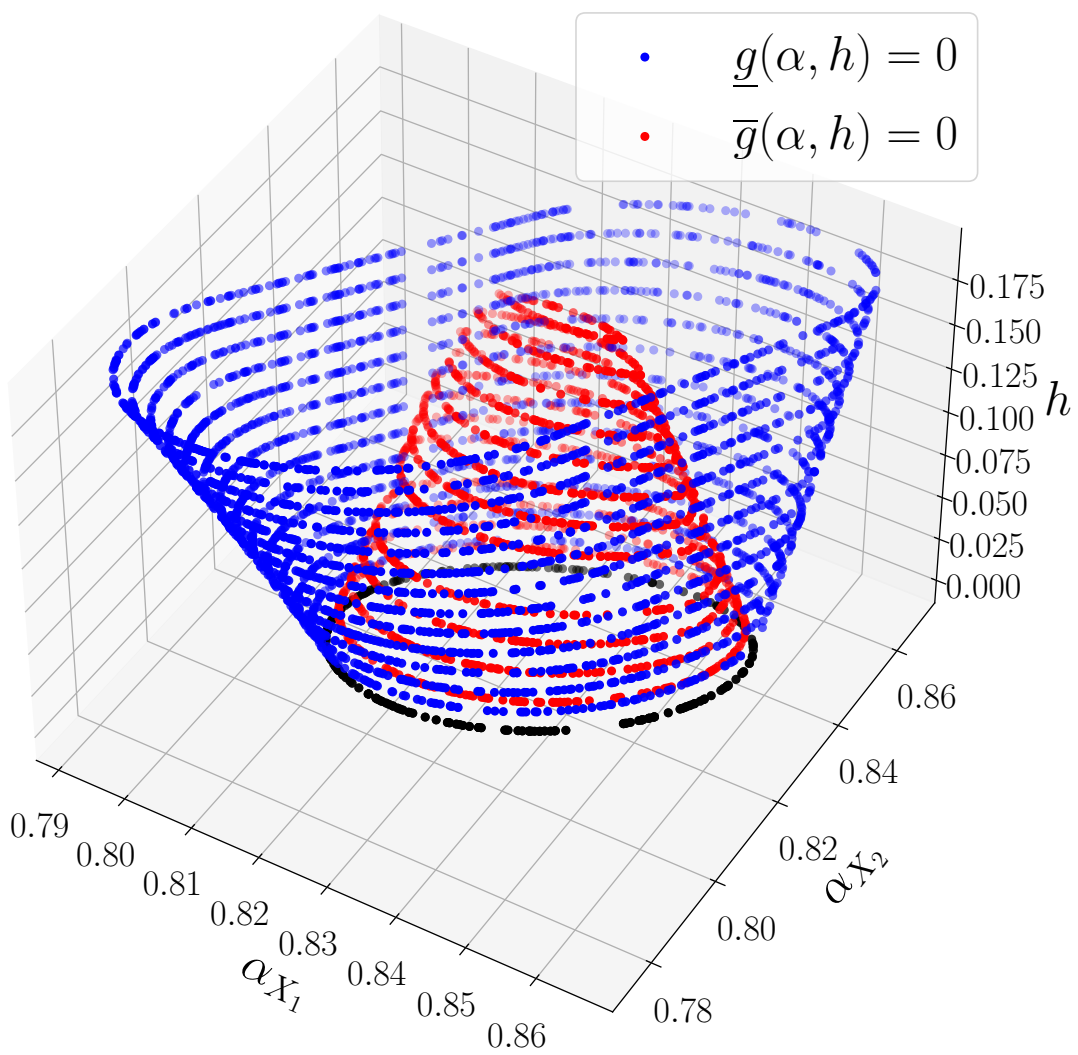

Figure 4: Illustration of limit-state surfaces $g(\boldsymbol{\alpha}, h)=0$ and $\bar{g}(\boldsymbol{\alpha}, h)=0$ for the interval representation for the Rosenbrock function.

$\mathbf{M}_{1}$ results. The isolines of the limit-state functions for the interval model on $Y$ are shown in Fig. 4, where the failure domain lies in the ellipsoid shape. Since, for a given $h, Y$ is a unique interval, its corresponding random set is the same interval and does not depend on $\alpha_{Y}$. Therefore, for a given $h$, the failure domain is a surface. The isolines are given at increasing horizons of uncertainty $h \in[0,0.19]$ which is why the plot is three-dimensional. One can see how $g(\boldsymbol{\alpha}, h)=0$ (used for $\overline{P_{\mathrm{f}}}$ estimation) gradually expands with $h$ while $\bar{g}(\boldsymbol{\alpha}, h)=0$ (used for $P_{\mathrm{f}}$ estimation) gradually reduces as expected.

Fig. 5 compares the isolines of the limit-state surfaces between the triangular and trapezoidal distributions at a given horizon level $h=0.19$. In this case, the dimension of $\boldsymbol{\alpha}$ is 3 which means that the failure domain is 


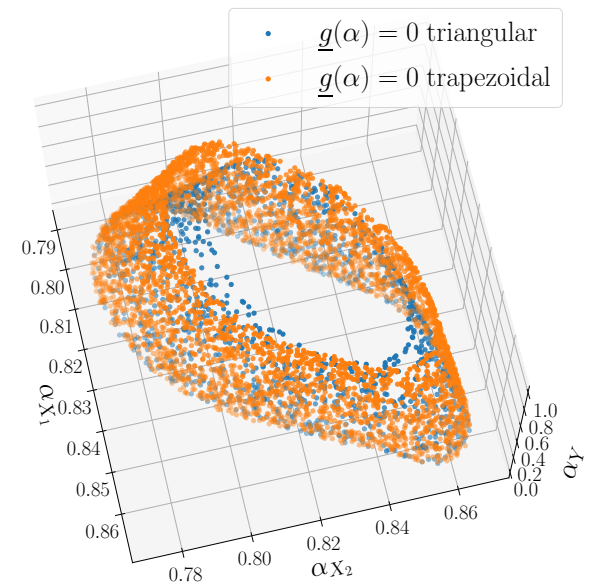

(a)

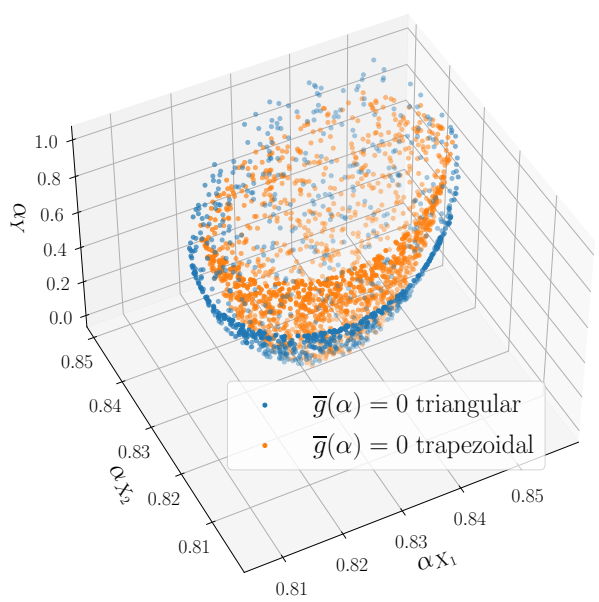

(b)

Figure 5: Comparison of $\underline{g}(\boldsymbol{\alpha})=0$ in (a) and $\bar{g}(\boldsymbol{\alpha})=0$ in (b) for the triangular and trapezoidal uncertainty models.

a volume which is why it is illustrated for a single value of $h$. For a given $\boldsymbol{\alpha}$, the corresponding random set induced by the triangular distribution is contained in the random set induced by the trapezoidal distribution. This explains why the failure volume obtained from the triangular model is contained in the one obtained from the trapezoidal model when considering the limit-state function $\underline{g}(\boldsymbol{\alpha}, h)=0$ while the opposite happens when considering the limit-state function $\bar{g}(\boldsymbol{\alpha}, h)=0$.

The analysis of the limit-state functions already gives a strong intuition on the inclusions of the bounds on the probability of failure obtained from the different uncertainty representations in $\mathbf{M}_{1}$. Fig. 7.(a) presents the robustness and opportuneness curves for the four different uncertainty models. The expected inclusions are obtained. One can notice that the extreme probabilities of failure remain close to the nominal result except for the lower probabilities of failure obtained with the interval model and considering RS theory (i.e., interval RS). This could be expected looking at Fig. 4 as the ellipses shrink considerably when the horizon of uncertainty grows. Nevertheless, computing the results of the interval model using the optimization method (i.e., interval DIRECT) yields very different results as the bounds on the probability of failure become very tight, even tighter than the bounds 


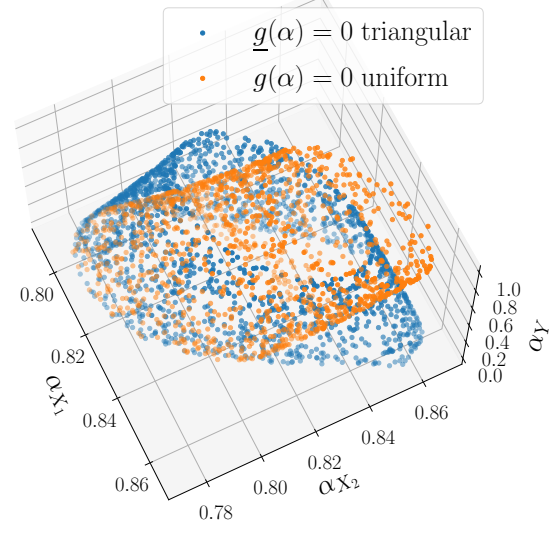

(a)

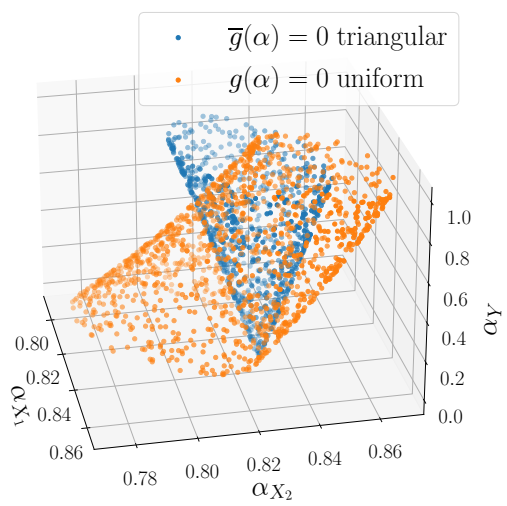

(b)

Figure 6: Comparison of $g(\boldsymbol{\alpha})=0$ in (a) and $\bar{g}(\boldsymbol{\alpha})=0$ in (b) for the triangular and uniform uncertainty models.

obtained from the trapezoidal model. This is probably caused by the strong non-linearity of the limit-state function.

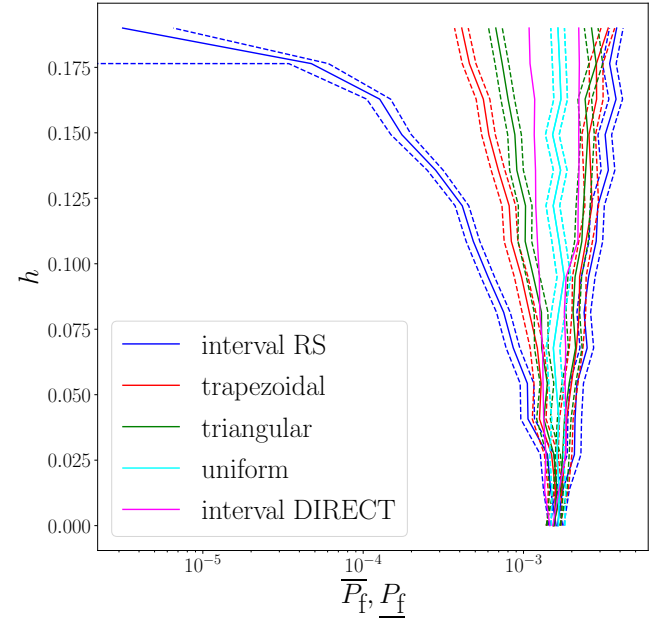

(a)

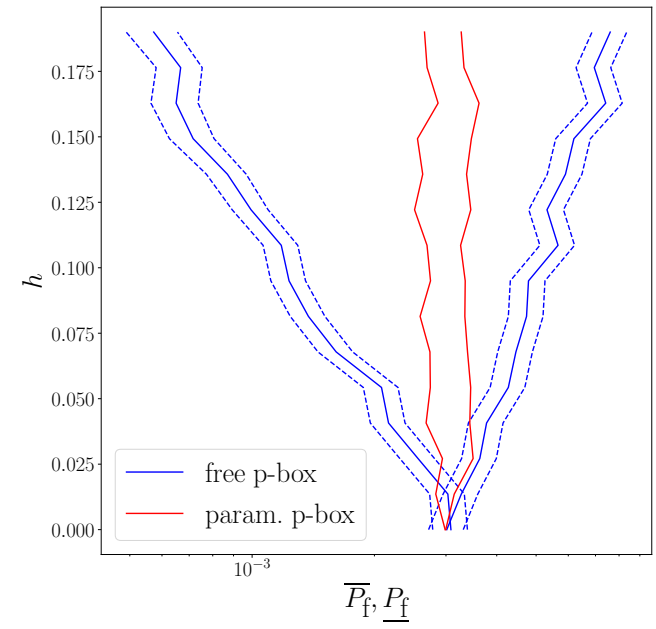

(b)

Figure 7: Robustness and opportuneness curves for the groups $\mathbf{M}_{1}$ (a) and $\mathbf{M}_{3}$ (b) for the Rosenbrock function. 
$\mathbf{M}_{3}$ results. Here, the free p-box and parametric p-box models are compared. It is recalled that, for an equivalent support, the parametric p-box model is more informative than the free p-box model which implies the bounds on the probability of failure of the second model to be contained in the bounds of the first model. The p-box models (free and parametric) are constructed by considering a Gaussian distribution on $Y: Y \sim \mathcal{N}\left(0.9, \sigma^{2}\right)$ with $\sigma \in[1-h, 1+h]$. The parametric p-box results are obtained by performing an optimization using the DIRECT algorithm on $\sigma$. Fig. 7.(b) presents the robustness and opportuneness curves. One can see a strong difference in behavior as the bounds induced by the parametric p-box model barely expand. This difference can again be explained by the strong non-linearity of the model.

$\mathbf{M}_{4}$ and $\mathbf{M}_{5}$ results. The $\mathbf{M}_{4}$ comparison aims, firstly, at numerically illustrating the relation between the triangular possibility distribution and its equivalent free p-box representation and, secondly, the link between the triangular possibility distribution and its discretized DS model. The $\mathbf{M}_{5}$ comparison aims at numerically illustrating the link between the probabilistic uniform cdf and its discretized DS model. Fig. 8 compares the limit-state functions between the triangular model and its equivalent p-box at $h=0.19$. It does seem that the limit-state functions of both representations have the same volume, though having a different shape. Note that, even if the different scales make it hard to see, the failure domain $\bar{g}(\boldsymbol{\alpha}) \leq 0$ is still included in the failure domain $\underline{g}(\boldsymbol{\alpha}) \leq 0$ for both representations as expected.

Fig. 9 compares the robustness and opportuneness curves for both comparisons and numerically confirms the expected results, despite the noise induced by the probability of failure estimations.

\subsection{Toy-case 2: a non-linear oscillator system}

The second toy-case corresponds to an adapted version of a two-degreeof-freedom oscillator as shown in Fig. 10 and seen in [30, 31]. The system is composed of two masses $m_{p}$ and $m_{s}$, two springs of stiffnesses $k_{p}$ and $k_{s}$, two damping ratios $\zeta_{p}$ and $\zeta_{s}$ and is subjected to a white noise base acceleration of intensity $S_{0}$. By denoting $F_{s}$ as the force capacity of the secondary spring, the reliability of the system is expressed through the following limit-state 


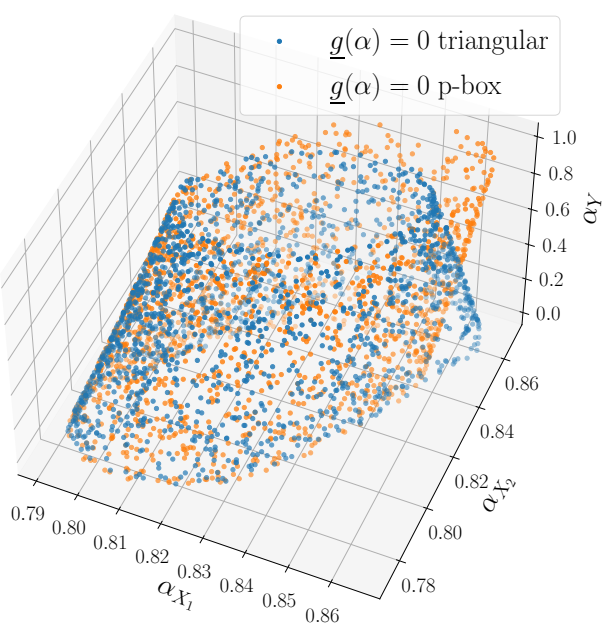

(a)

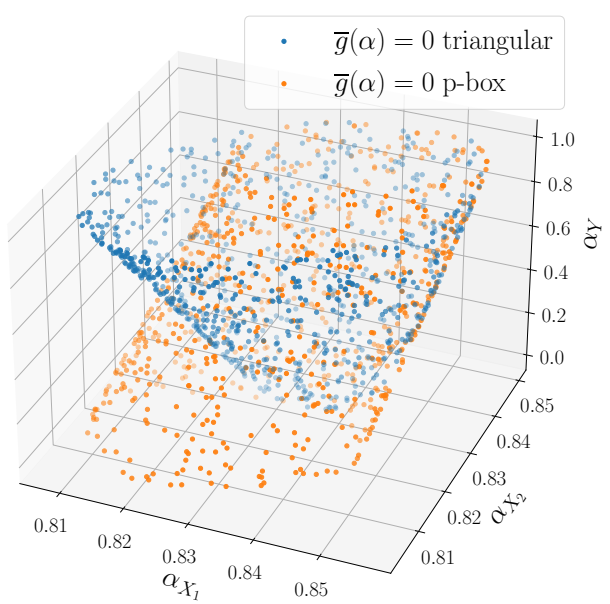

(b)

Figure 8: Comparison of $g(\alpha)=0$ in (a) and $\bar{g}(\alpha)=0$ in (b) for the triangular and triangular-pbox uncertainty models.

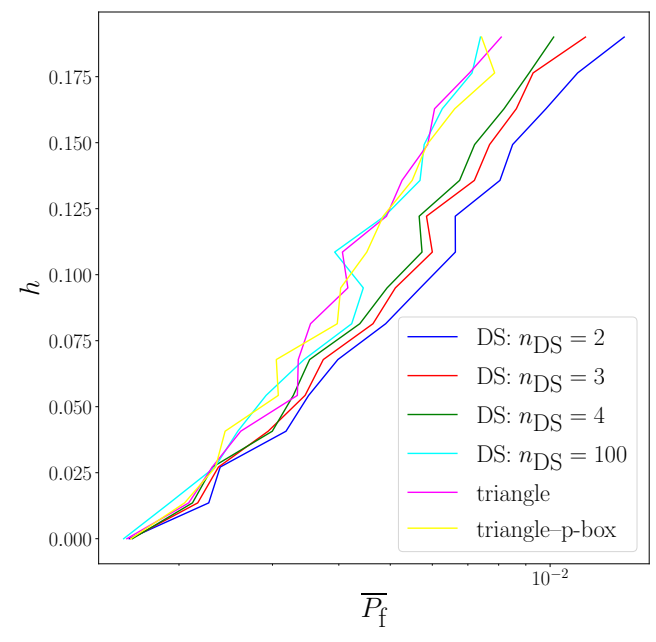

(a)

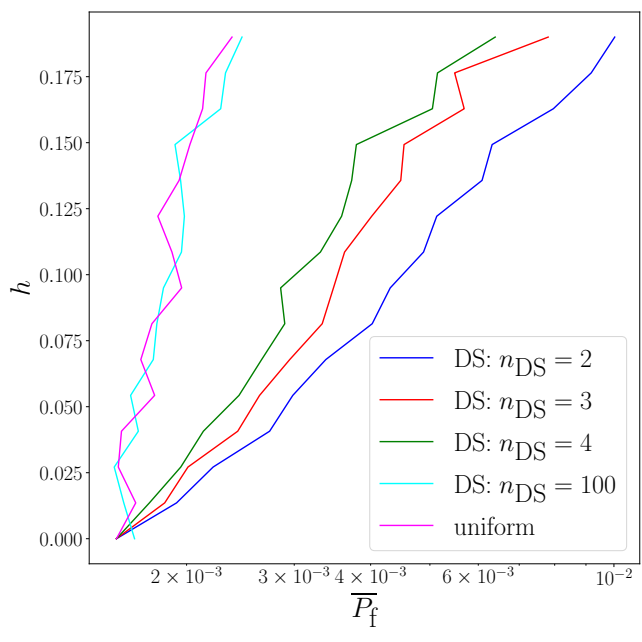

(b)

Figure 9: Robustness curves for the groups $\mathbf{M}_{4}$ (a) and $\mathbf{M}_{5}$ (b) for the Rosenbrock function.

454

$$
\begin{aligned}
& \text { function: } \\
& \qquad g(\mathbf{X}, \mathbf{Y})=F_{s}-3 k_{s} \sqrt{\frac{\pi S_{0}}{4 \zeta_{s} \omega_{s}^{3}}\left[\frac{\zeta_{a} \zeta_{s}}{\zeta_{p} \zeta_{s}\left(4 \zeta_{a}^{2}+r^{2}\right)+\gamma \zeta_{a}^{2}} \frac{\left(\zeta_{p} \omega_{p}^{3}+\zeta_{s} \omega_{s}^{3}\right) \omega_{p}}{4 \zeta_{a} \omega_{a}^{4}}\right]}
\end{aligned}
$$


where $\gamma=m_{s} / m_{p}$ is the mass ratio, $\omega_{p}=\left(k_{p} / m_{p}\right)^{1 / 2}$ and $\omega_{s}=\left(k_{s} / m_{s}\right)^{1 / 2}$ the natural frequencies, $\omega_{a}=\left(\omega_{p}+\omega_{s}\right) / 2$ the average frequency ratio, $\zeta_{a}=$ $\left(\zeta_{p}+\zeta_{s}\right) / 2$ the average damping ratio and $r=\left(\omega_{p}-\omega_{s}\right) / \omega_{a}$ a tuning parameter. The random vector $\mathbf{X}$ gathers $n_{X}=3$ independent random variables whose probabilistic modeling is given in Table 2. The epistemic vector $\mathbf{Y}$ is of dimension $n_{Y}=5$ and its epistemic characteristics are given in Table 3. One supposes here that nominal values are known for the stiffnesses, the damping ratios and the force capacity.

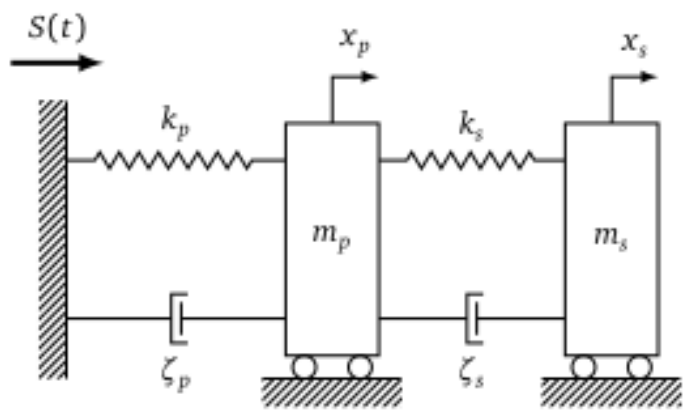

Figure 10: Two-degree-of-freedom damped oscillator.

The horizon of uncertainty is discretized into 10 values in [0,0.05]. The groups of comparison that are studied are $\mathbf{M}_{1}$ and $\mathbf{M}_{2}$.

Table 2: Input probabilistic modeling of $\mathbf{X}$.

\begin{tabular}{llll}
\hline Variable $X_{i}$ & Distribution & Mean $\mu_{X_{i}}$ & $\delta$ \\
\hline$X_{1}=m_{p}(\mathrm{~kg})$ & Lognormal & 1.5 & $10 \%$ \\
$X_{2}=m_{s}(\mathrm{~kg})$ & Lognormal & 0.01 & $10 \%$ \\
$X_{3}=S_{0}\left(\mathrm{~m} . \mathrm{s}^{-2}\right)$ & Lognormal & 100 & $10 \%$ \\
\hline
\end{tabular}

$\mathbf{M}_{1}$ results. Before showing the robustness and opportuneness curves for all representations, Fig. 11 compares these curves for the trapezoidal representation estimated using the FORM-IS and Subset Simulation algorithms. The curves obtained by evaluating the hybrid limit-state functions using the vertex method [32] (which states that the extreme values of the limit-state function are obtained at combinations of the extreme values of $Y_{i}$ ) instead of 
Table 3: Epistemic characteristics of $\mathbf{Y}$.

\begin{tabular}{ll}
\hline Variable $Y_{i}$ & $\widetilde{Y}_{i}$ \\
\hline$Y_{1}=k_{p}\left(\mathrm{~N} . m^{-1}\right)$ & 1 \\
$Y_{2}=k_{s}\left(\mathrm{~N} . \mathrm{m}^{-1}\right)$ & 0.01 \\
$Y_{3}=\zeta_{p}(1)$ & 0.05 \\
$Y_{4}=\zeta_{s}(1)$ & 0.02 \\
$Y_{5}=F_{s}(\mathrm{~N})$ & 11 \\
\hline
\end{tabular}

an optimization algorithm are also given in the same figure. The curves suggest a high confidence in the results obtained with the FORM-IS algorithm and seem to confirm the hypothesis introduced with the vertex method.

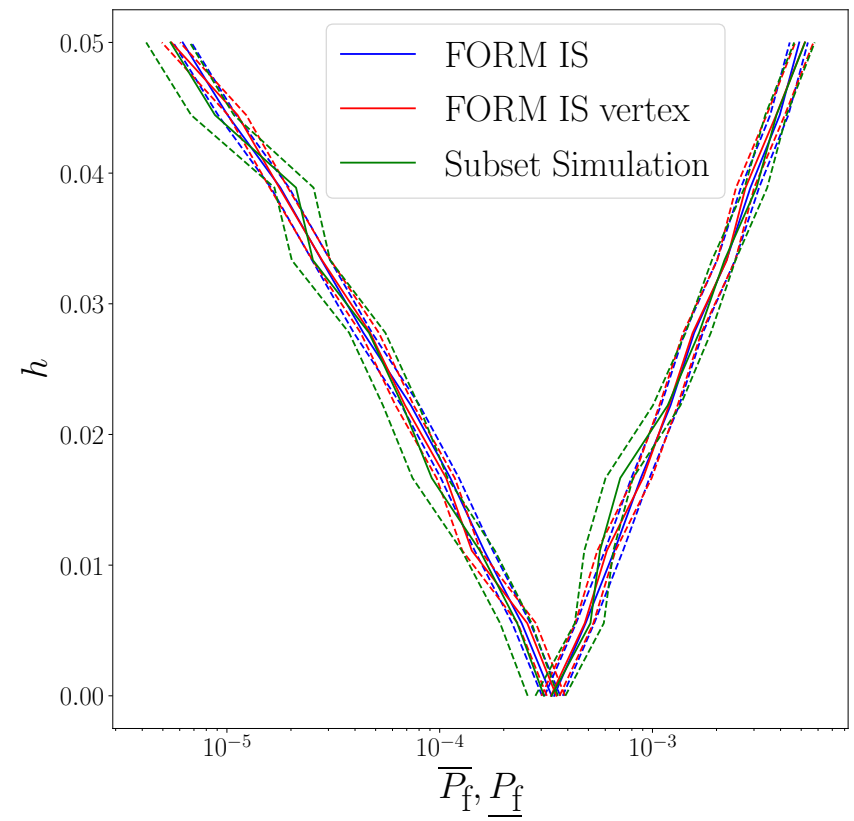

Figure 11: $P_{\mathrm{f}}$ estimators comparison for the oscillator case.

473

The robustness and opportuneness curves are given in Fig. 12.(a) and the corresponding VoI surface plot in Fig. 12.(b). Once again, the minimum probability of failure with the interval-RS model quickly decreases compared to the other representations including the interval-DIRECT model. This is not so much the case for the maximum probability of failure for which 
the highest values of $R_{\bar{P}_{f}}$ are obtained from the triangle representation to the uniform representation. Fig. 13.(a) gives the sensitivity measures from

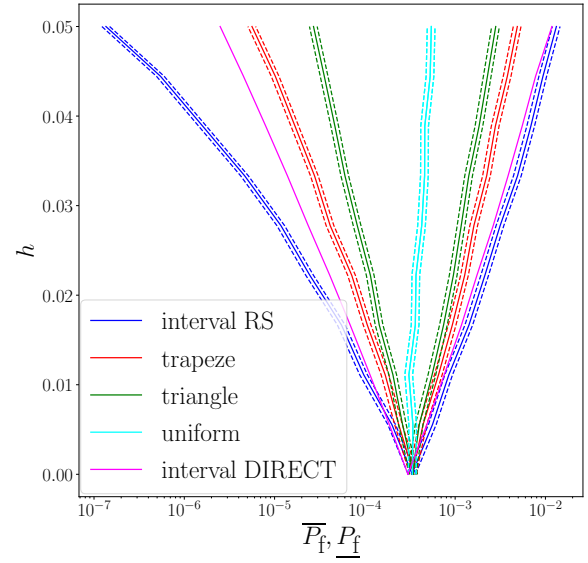

(a)

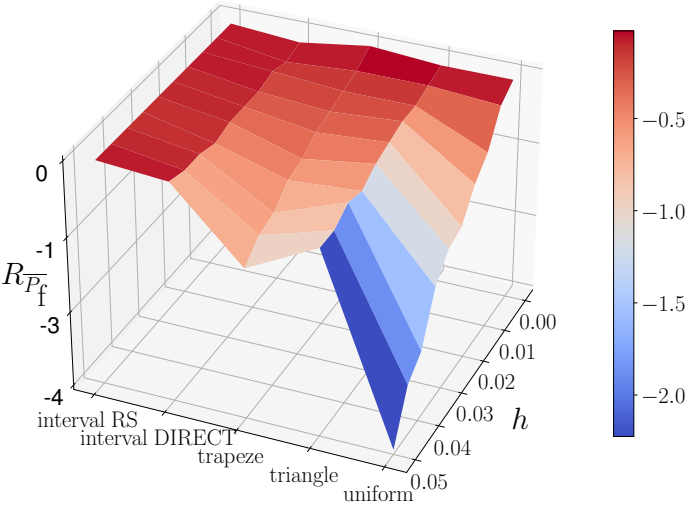

(b)

Figure 12: Robustness and opportuneness curves (a) and VoI metric (b) considering the $\mathbf{M}_{1}$ group for the non-linear oscillator case.

the interval to the trapezoidal models and Fig. 13.(b) gives the sensitivity measures from the trapezoidal to the triangular models. It appears that the added information in $F_{s}$ has, for both cases, the strongest influence on the robustness of the probability of failure even if it seems that $k_{p}$ also has a strong influence.

$\mathbf{M}_{2}$ results. Here, the multi-parallelepiped uncertainty model is used to model the epistemic vector by considering an equal coefficient of linear correlation $\rho_{k_{p} \zeta_{p}}=\rho_{k_{s} \zeta_{s}}=\rho$. Fig. 14 presents the robustness and opportuneness curves for different values of the coefficient of correlation. The $95 \%$ confidence intervals are not depicted for the sake of clarity. As expected, the higher the coefficient of correlation in terms of absolute value, the narrower the bounds on the probability of failure. Nevertheless, the bounds will shrink significantly as soon as a non-zero coefficient of correlation is given but the results between a low or high coefficient do not considerably differ. 


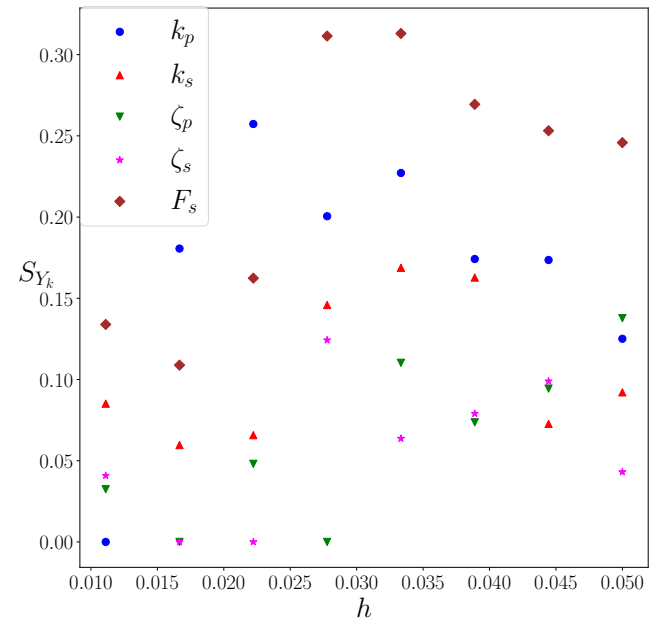

(a)

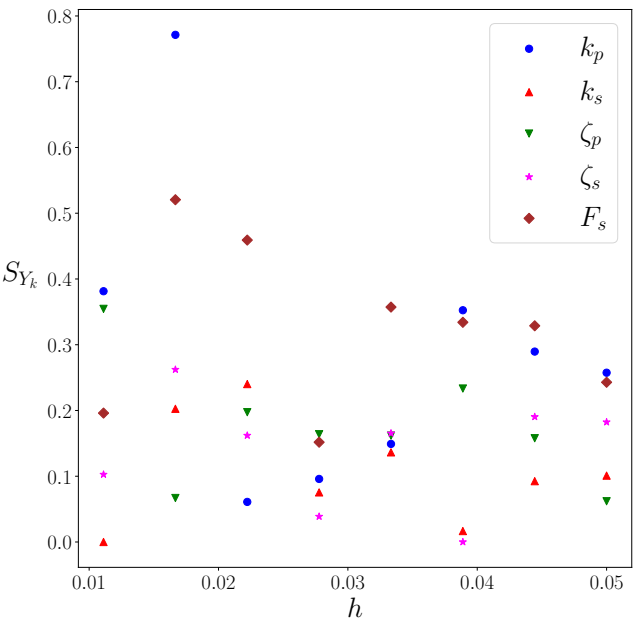

(b)

Figure 13: Sensitivity analysis interval-trapezezoidal (a) and sensitivity analysis trapezoidal-triangular (b) for the oscillator case.

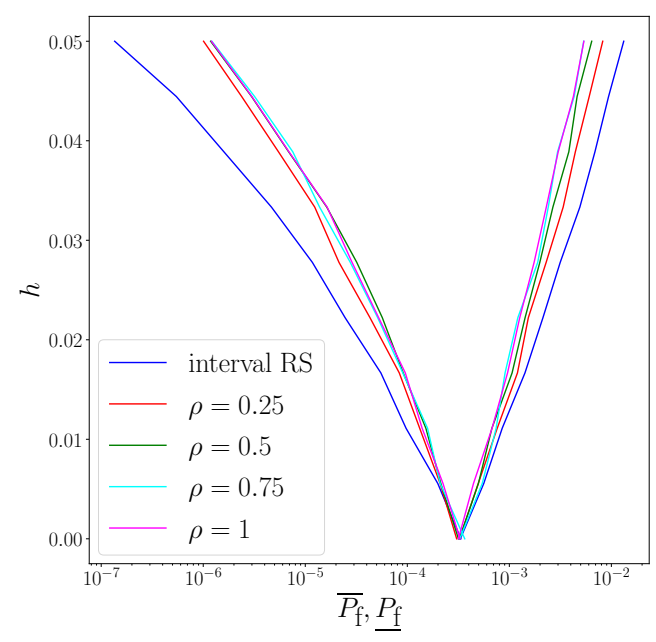

(a)

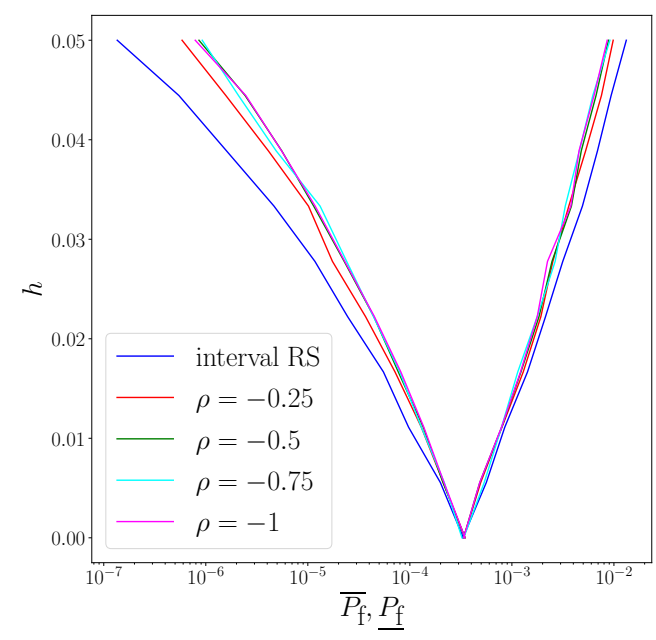

(b)

Figure 14: $\mathbf{M}_{2}$ comparison for positive coefficients of correlation (a) and $\mathbf{M}_{2}$ comparison for negative coefficients of correlation (b)

\subsection{Industrial use-case: reliability assessment of penstocks}

In this section, the methodology is applied to an industrial use-case relevant to the French electricity company EDF which concerns the reliability 
study of penstocks [33, 34]. Note that this industrial application is illustrative: the choice of epistemic variables should be further substantiated. It is meant to demonstrate the feasibility of the methodology on a more complex industrial use-case. EDF operates more than 500 penstocks having a total length of over $300 \mathrm{~km}$. Penstocks are pipes made of steel used to transport water under pressure from the water dam to the hydroelectric turbine. Due to thickness loss resulting from corrosion, their mechanical integrity must be justified. The usual justification relies on diagnoses involving thickness measurements and the evaluation of a deterministic margin factor MF.

To optimize MF, a general reliability approach was developed to assess annual probabilities of failure at year $N$ of a given penstock. Two failure modes have been investigated: plastic collapse (parent metal) and brittle failure (welds), due to the presence of cracks appearing during the welding process. In the present application, only the second failure mode is considered since its reliability analysis is more complex:

- the limit-state function is locally non-differentiable and can be discontinuous;

- the annual probability of failure estimated here is a conditional probability considering that the penstock passed a hydraulic test after its production in the workshop.

The conditional probability at year $N$ can be expressed as:

$$
P_{\mathrm{f}}=\operatorname{Pr}\left(G_{N} \cap G_{N-1}<0 \mid G_{\mathrm{ht}} \geq 0\right)=\frac{\operatorname{Pr}\left(G_{N} \cap G_{N-1} \leq 0 \cap G_{\mathrm{ht}} \geq 0\right)}{\operatorname{Pr}\left(G_{\mathrm{ht}} \geq 0\right)}
$$

where $G_{N}$ is the limit-state function at year $N, G_{N-1}$ the limit-state function at year $N-1$ and $G_{\mathrm{ht}} \geq 0$ is the event that the penstock successfully passed the hydraulic test. As the G-function decreases over time due to the monotonic corrosion degradation, an equivalent expression is as follows:

$$
P_{\mathrm{f}}=\operatorname{Pr}\left(G_{N} \times G_{N-1}<0 \mid G_{\mathrm{ht}} \geq 0\right)=\frac{\operatorname{Pr}\left(G_{N} \times G_{N-1} \leq 0 \cap G_{\mathrm{ht}} \geq 0\right)}{\operatorname{Pr}\left(G_{\mathrm{ht}} \geq 0\right)}
$$

In this work, the last expression will be preferred as it is better to reduce the number of intersecting events (from 3 to 2). The expressions of $G_{N}$, $G_{N-1}$ and $G_{h t}$ are analytical but depend on too many parameters to be detailed here except for the parameters considered in the probabilistic and 
epistemic vectors. The failure criterion corresponds to the failure assessment diagram given in [35]. The probabilistic vector $\mathbf{X}$ is of dimension $n_{X}=5$ and its characteristics are given in Table 4 where $R_{m}$ is the ultimate tensile strength, $\Delta e_{\text {extra }}$ the extra thickness added to the design thickness, $\Delta e_{\text {corr }}$ the thinning due to water and atmospheric corrosion, $\varepsilon$ a parameter used to linearly express the yield strength in function of $R_{m}$ and $K_{I C}$ the tenacity of the material.

Table 4: Input probabilistic modeling of $\mathbf{X}$ for the penstock use-case.

\begin{tabular}{lllll}
\hline Variable $X_{i}$ & Distribution & Param 1 & Param 2 & Param 3 \\
\hline$X_{1}=R_{m}(\mathrm{MPa})$ & Lognormal & 480 & 24 & - \\
$X_{2}=\Delta e_{\text {extra }}(\mathrm{mm})$ & Normal & 0 & 0.25 & - \\
$X_{3}=\Delta e_{\text {corr }}(\mathrm{mm})$ & Normal & 1 & 0.4 & - \\
$X_{4}=\varepsilon(\mathrm{MPa})$ & Normal & 0 & 16.816 & - \\
$X_{5}=K_{I C}(\mathrm{MPa} \cdot \sqrt{\mathrm{m}})$ & Weibull Min & 90 & 4 & 20 \\
\hline
\end{tabular}

The epistemic vector $\mathbf{Y}$ is of dimension $n_{Y}=3$ and its characteristics are given in Table 5 where $e_{\text {nom }}$ is the nominal thickness of the pipe, $\Delta e_{\text {an }}$ the annual loss of thickness and $a$ the height of the crack. It should be noted that the values of the parameters of the probabilistic and epistemic variables were chosen in a large panel of values that represent the variety of all the penstocks operated by EDF in order to have an industrially relevant type of penstock which has a nominal probability of failure between $10^{-9}$ and $10^{-8}$.

Table 5: Epistemic characteristics of $\mathbf{Y}$ for the penstock use-case

\begin{tabular}{ll}
\hline Variable $Y_{i}$ & $\widetilde{Y}_{i}$ \\
\hline$Y_{1}=e_{\text {nom }}(\mathrm{mm})$ & 8 \\
$Y_{2}=\Delta e_{\text {an }}(\mathrm{mm})$ & 0.06 \\
$Y_{3}=a(\mathrm{~mm})$ & 2 \\
\hline
\end{tabular}

The standard reliability analysis containing uniquely probabilistic variables is performed using FORM-IS with OpenTURNS. Other techniques could also have been used such as Subset Simulation. It should be pointed out that calculating a conditional probability using RS theory is not as straightforward as in Eqs. (15.a) and (15.b). Indeed, one cannot express in a trivial way the maximum or the minimum of the probability of failure as a function 
of the maximum or minimum of both limit-states $G_{N} \times G_{N-1}$ and $G_{\mathrm{ht}}$. Nevertheless in this case, as $\mathbf{Y}$ has a greater impact on $G_{N} \times G_{N-1}$ than $G_{\mathrm{ht}}$, the following simplification is performed:

$$
\bar{P}_{\mathrm{f}} \approx \frac{\operatorname{Pr}\left[\min _{\Gamma(\boldsymbol{\alpha})}\left(G_{N} \times G_{N-1}\right) \leq 0 \cap G_{\mathrm{ht}}\left(\boldsymbol{\alpha}^{*}\right) \geq 0\right]}{\operatorname{Pr}\left[G_{\mathrm{ht}}\left(\boldsymbol{\alpha}^{*}\right) \geq 0\right]}
$$

where $\boldsymbol{\alpha}^{*}=\arg \min \left(G_{N} \times G_{N-1}\right)$. In this industrial use-case, only the maximum probability of failure $\bar{P}_{\mathrm{f}}$ will be of interest. The results of the groups of comparison $\mathbf{M}_{1}, \mathbf{M}_{2}$ and $\mathbf{M}_{3}$ are given in the following.

$\mathbf{M}_{1}$ results. The FORM-IS algorithm is, once again, the first choice to estimate the probabilities. Nevertheless, it is necessary to verify the results with another algorithm. Fig. 15 compares the robustness curves obtained with the FORM-IS and the Subset Simulation algorithms considering the interval model on $\mathbf{Y}$. Despite being less smooth, the results obtained with the Subset Simulation algorithm are very similar to those obtained with FORM-IS.

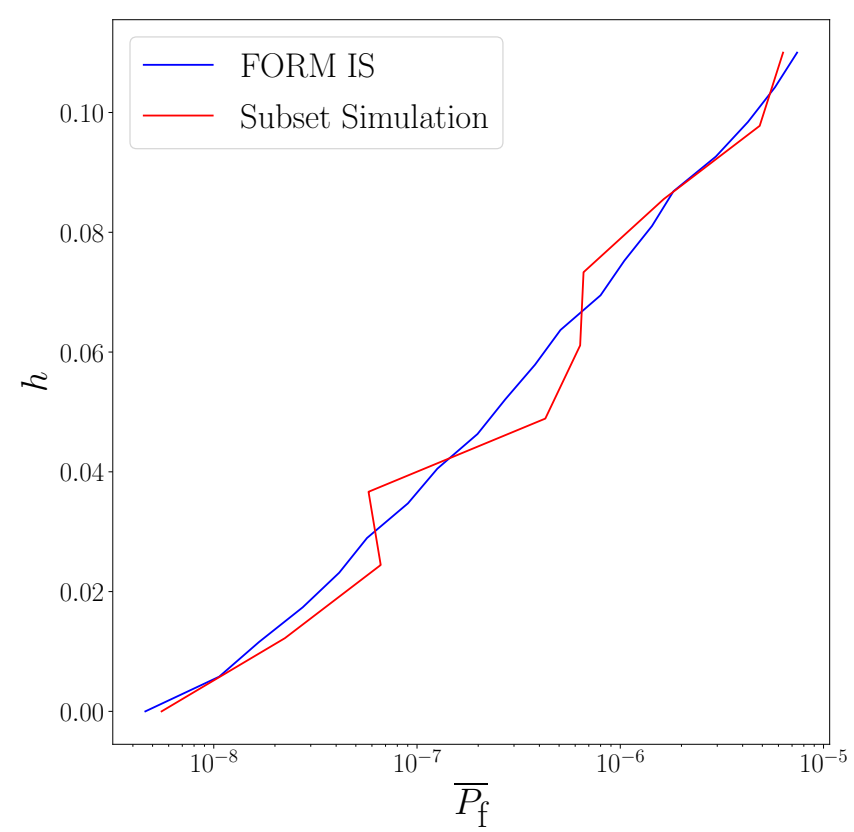

Figure 15: $P_{\mathrm{f}}$ estimators comparison for the penstock use-case.

Fig. 16.(a) shows the robustness curves of $\mathbf{M}_{1}$ and Fig. 16.(b) shows the corresponding VoI surface plot of the VoI metric $R_{\bar{P}_{\mathrm{f}}}$. Once again, the 


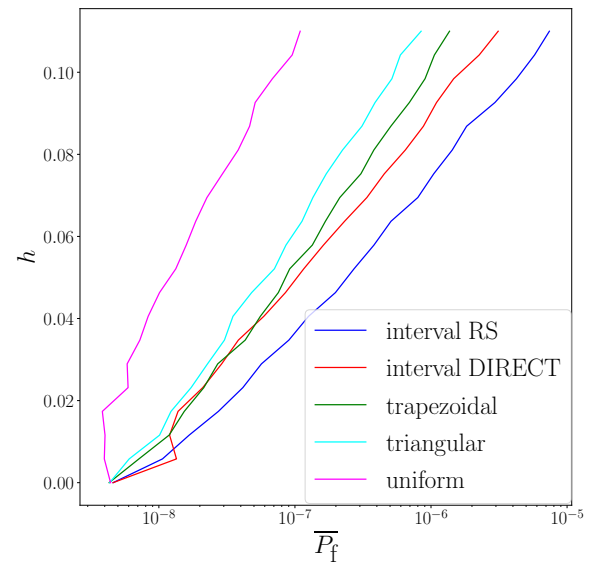

(a)

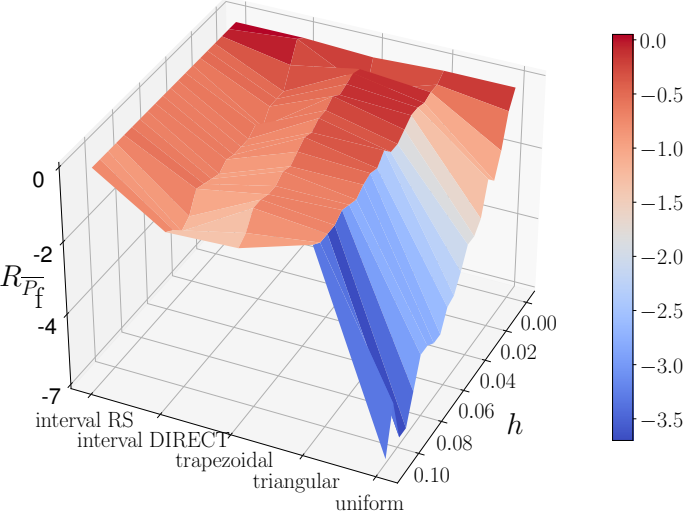

(b)

Figure 16: Robustness curves (a) and VoI metric (b) considering the $\mathbf{M}_{1}$ group for the penstock use-case.

probabilistic information of a uniform distribution considerably reduces the maximum probability of failure and therefore improves the robustness. The difference between both interval results is also quite significant.

Fig. 17 presents the sensitivity results from the interval to the trapezoidal representations. The classification of the most influential variables on the information is quite clear whatever the horizon of uncertainty as the added information on $e_{\text {nom }}$ has a strong influence, on $K_{I C}$ a non-negligible influence and on $\Delta e_{\text {an }}$ a very weak influence.

$\mathbf{M}_{2}$ results. Fig. 18.(a) presents the results with the multi-parallelepiped model by considering a coefficient of correlation $\rho=\rho_{Y_{1} Y_{2}}$ between $e_{\text {nom }}$ and $\Delta e_{\text {an }}$. It seems that a negative coefficient of correlation has no impact on the robustness while a positive coefficient has a very limited impact.

$\mathbf{M}_{3}$ results. The p-box model is constructed by considering a Gaussian distribution for $Y_{i}: Y_{i} \sim \mathcal{N}\left(\widetilde{Y}_{i}, \sigma_{i}^{2}\right)$ with $\sigma_{i} \in\left[\widetilde{\sigma}_{i}(1-h), \widetilde{\sigma}_{i}(1+h)\right]$ and $\widetilde{\sigma}=[0.4,0.003,0.1]^{\top}$. For this case, the horizon of uncertainty belongs to $h \in[0,0.5]$. The parametric p-box results are still obtained by performing 


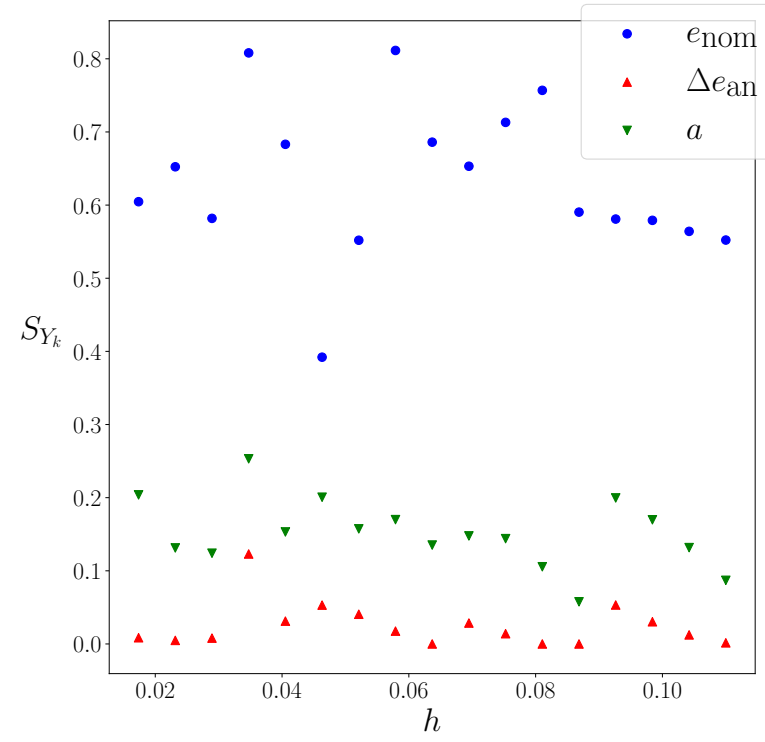

Figure 17: Sensitivity analysis interval-trapezezoidal for the penstock use-case.

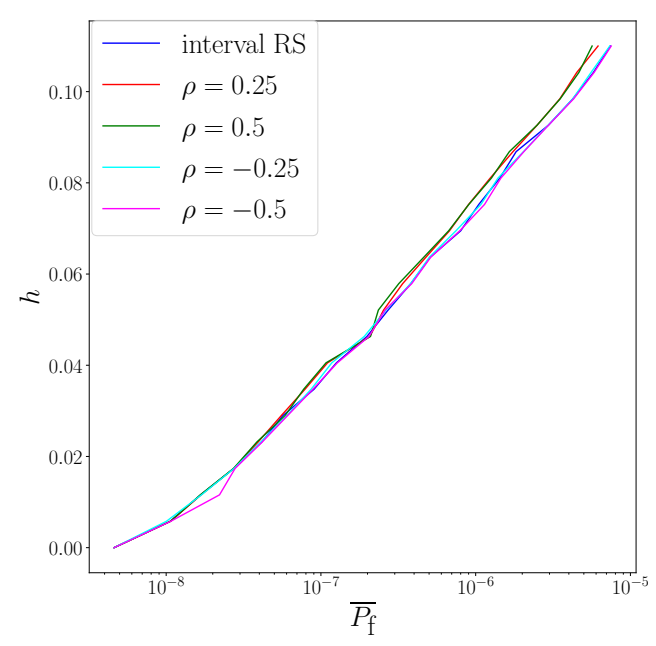

(a)

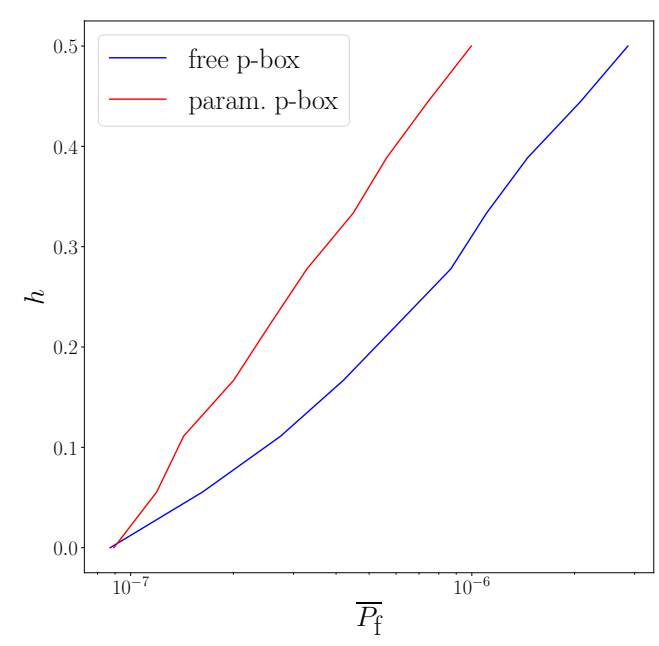

(b)

Figure 18: Robustness curves for the groups $\mathbf{M}_{2}$ (a) and $\mathbf{M}_{3}$ (b) for the penstock use-case.

577 an optimization using the DIRECT algorithm on $\sigma$. Fig. 18.(b) presents the 578 robustness curves for both representations. The added information in the 579 parametric p-box improves the robustness as expected. 


\section{Discussions}

This section aims in a first place at summarizing all the results from above with respect to the objective of this work which is to analyse the effect of different models of uncertainty on a robustness analysis in the context of HRA. The use-cases enable to numerically translate the links presented in Fig. 1 by constructing and comparing opportuneness and robustness curves. These links, and therefore the comparison, are divided into two main groups. The first one, which involves the comparison groups $M_{1}, M_{2}$ and $M_{3}$, shows in what extent a more informative uncertainty model may affect robustness and opportunity. Indeed, adding information will often lead to a gain in robustness (the model can tolerate more uncertainty) and a loss in opportunity (more uncertainty is needed for a positive unexpected outcome). In the context of HRA, this notion is seen as a narrowing of the bounds of the probability of failure until reaching a unique value for purely probabilistic or deterministic models. The more the support of the epistemic variables grows the more narrowing is observed. On the other hand, the more informative a model is the more dependent the quantity of interest is on the hypothesis made. Nevertheless, the benefits of acquiring information strongly depend on the decision-making context. Indeed, in the case of safety assessment for which very small probabilities of failure are estimated, a gain of information is way more valuable if it affects the robustness curve rather than the opportuneness curve.

The behavior of the numerical model (i.e., the limit-state functions in the case of HRA) with respect to the epistemic variables also has a key role on the value of information. For example, a monotonous behavior will yield the same bounds on the probability of failure whether free or parametric p-boxes are considered. A reduced convex set (i.e., more informative) will have no impact on the robustness curve if the worst performance was initially obtained at a point that is still contained in the more informative set. In most practical cases, such information may unfortunately only be available after the robustness analysis.

The second main group concerns the comparison groups $M_{4}$ and $M_{5}$ which emphasize some links between different uncertainty models in the framework of this paper. More precisely, it numerically confirms that possibility distributions and DS structures may also be seen as free p-boxes and that probability and possibility distributions may be considered as DS structures (with a loss of information that depends on the discretization process). This is 
interesting for two main reasons. The first one is related to the fact that all these uncertainty models with different interpretations and mathematical foundations may be intimidating for an average decision maker. These links show how different models can be closely related in the context of HRA. The second reason is that it enables to widen the use of smart numerical methods initially established for a specific uncertainty framework such as p-boxes for which a lot of content is proposed.

Additionally, this section wants to clarify the use of the proposed methodology. The info-gap framework is initially built for taking robust decisions in the context of strong uncertainty. The present work does certainly not aim at ranking any uncertainty representation nor does it want to emphasize the proposed methodology for performing an info-gap analysis. The choice of an uncertainty model strongly depends on the available information and on the context in which a decision must be made (e.g., a safety requirement). The reader is referred to [16] for more insights into the use of the different uncertainty representations. The info-gap method brings an additional tool for confronting a decision to the hypotheses that were made in order to take that decision. Therefore, it is complementary with the probabilistic framework for decisions based on reliability quantities. The possible combination of the different uncertainty models considered through random set theory together with the info-gap framework offers a wide range of possibilities for conducting a suitable robustness analysis on reliability quantities.

For example, in this work, the horizon of uncertainty is applied to the supports of the epistemic variables for comparison purposes. In some applications, the support may be fixed but the uncertainty representation challenged. One may consider a nominal precise cumulative distribution function that becomes a less informative DS structure (or wider p-box) at increasing horizons of uncertainty until finally reaching the interval model. The way of performing info-gap remains partially subjective as choices are made by the decision-maker. Our idea is to face uncertainty the most objectively in order to take a trustworthy decision.

\section{Conclusion}

In this paper, a methodology was proposed in order to analyse the robustness of the upper bound of a probability of failure with respect to different epistemic uncertainty representations in input. In the context of hybrid reliability analysis, the random set framework is suitable to model and propagate 
different representations of uncertainty to estimate reliability quantities of interest such as bounds on a probability of failure. An info-gap robustness analysis was performed by considering each type of uncertainty model in an increasing support of the epistemic variables.

This methodology enabled to compare robustness and opportuneness curves between uncertainty models that are more or less informative for two academic examples and one industrial use-case related to the reliability assessment of hydraulic penstocks. As expected, it is seen that increasing the support of the epistemic variables leads to increasing the effect of the choice of the uncertainty model on the bounds of the probability of failure and therefore on the robustness analysis. The objective of this study is not to determine the best representation of uncertainty, as this depends on the available information, but to provide insights about the impact (in terms of robustness) of the uncertainty model.

Such comparison is limited to relatively simple implementations of the different components involved whether it is for the uncertainty models, the use-cases or the application of info-gap. Moreover, the case of dependencies within the random and epistemic variables and between both of them was omitted. This is contradictory with the fact of confronting strong hypothesis with the info-gap method. Finally, no comparison is given in terms of function evaluations. Yet, hybrid reliability analysis combined to info-gap requires a huge computational effort when no specific hypotheses are made as it demands a very large number of evaluations of the initial limit-state function. The question of feasibility with complex numerical codes should also be part of the analysis.

Future work will compare the computational effort required as a function of the uncertainty model used in a robustness analysis. This comparison will depend on many factors and especially on hypotheses that are made (for example, the monotony of the limit-state function with respect to the epistemic variables), and the numerous strategies that have already been developed to reduce the computational burden (for example, combination of surrogate models with smart optimization algorithms).

\section{Appendix A. Standard reliability analysis}

The performance $z \in \mathbb{R}$ (assumed to be a scalar here for the sake of simplicity) of a system is evaluated via an analytical or numerical model $\mathcal{M}(\mathbf{x})$ where $\mathbf{x} \in \mathbb{R}^{n_{X}}$ with $n_{X}$ the number of input variables. By considering 
the convention that the performance must not exceed a given threshold $z_{\text {th }} \in$ $\mathbb{R}$, the limit-state function $g(\cdot)$ is defined such that:

$$
g(\mathbf{x})=z_{\mathrm{th}}-\mathcal{M}(\mathbf{x}) .
$$

It follows the definition of the failure domain $\mathcal{F}$ :

$$
\mathcal{F}=\left\{\mathbf{x} \in D_{\mathbf{X}}, g(\mathbf{x}) \leq 0\right\}
$$

with $\mathcal{F}^{0}=\left\{\mathbf{x} \in D_{\mathbf{X}}, g(\mathbf{x})=0\right\}$ the limit-state surface. In order to determine whether the system lies in the failure or safety domain, the uncertain input variables $x_{i}\left(i=1, \ldots, n_{X}\right)$ are modeled using the probabilistic framework. The input vector is considered as a realization of the random vector $\mathbf{X}=$ $\left(X_{1}, X_{2}, \ldots, X_{n_{X}}\right)^{\top}$ to which a supposedly known joint probability density function (pdf) $f_{\mathbf{X}}$ is attributed. One may then calculate a reliability quantity of interest such as the probability of failure $P_{\mathrm{f}}$ defined as:

$$
P_{\mathrm{f}}=\operatorname{Pr}[g(\mathbf{X}) \leq 0]=\int_{\mathcal{F}} f_{\mathbf{X}}(\mathbf{x}) d \mathbf{x}=\int_{\mathbb{R}^{n} X} \mathbb{1}_{\mathcal{F}}(\mathbf{x}) d \mathbf{x}
$$

where $\mathbb{1}_{\mathcal{F}}$ is the indicator function that is equal to one when the event $\mathbf{x} \in \mathcal{F}$ is realized and zero when it is not. Several techniques exist to evaluate Eq. (A.3) such as sampling methods [24] or approximation methods [26].

\section{Appendix B. Uncertainty models}

Interval model. The interval representation only uses bounds to model the uncertainty on an input quantity $Y_{i}$. Therefore, the only hypothesis made here is that $Y_{i}$ belongs to the interval $I_{Y_{i}}=\left[Y_{i}^{L}, Y_{i}^{U}\right]$ with $Y_{i}^{L}$ the lower bound and $Y_{i}^{U}$ the upper bound. Any value within the interval can be taken without any assumption about the fact that values are more likely than others. Note that it is totally different than assigning a uniform distribution over the interval since a uniform distribution is still a particular distribution and assumes an existing measure. When each $Y_{i}$ is represented as an interval, the input space becomes the $n_{Y}$-box represented by the Cartesian product $I_{Y}=\times_{i=1}^{n_{Y}} I_{Y_{i}}$ where $n_{Y}$ is the number of interval variables. After propagation through the numerical model $\mathcal{M}(\cdot)$, the performance is also an interval with no additional information. The bounds $\left[Z^{L}, Z^{U}\right]$ may be estimated using an optimization algorithm or the vertex method which states that the extreme values of the performance are obtained for combinations of the extreme values of $Y_{i}$. Methods to treat hybrid reliability problems involving both random and interval variables can be found in $[36,37]$. 
Convex model. Convex models [11] are also a non-probabilistic representation of uncertainty which contains the interval model. It enables to add information concerning possible dependencies between the input variables. The ellipsoid and the parallelogram convex models are common examples. When the input variables are independent, the convex model reduces to the $n_{Y^{-}}$ box which characterizes the interval representation. In the same way as for the interval model, the bounds on the performance function can be obtained using an optimization algorithm in the convex set. The multi-parallelepiped model [38] is used in this paper as it has the advantage of combining dependent and independent variables. Moreover, a sample in this convex set can be obtained from a sample $\mathbf{u}=\left(u_{1}, \cdots, u_{n_{Y}}\right)^{\top}$ of the hypercube $U=[-1,1]^{n_{Y}}$ with the following transformation:

$$
Y_{i}=\frac{Y_{i}^{W}}{\sum_{j=1}^{n_{Y}}|\rho(i, j)|} \sum_{k=1}^{n_{Y}} \rho_{i k} u_{k}+Y_{i}^{C}, i=1,2, \ldots, n_{Y}
$$

where $Y_{i}^{C}=\frac{Y_{i}^{U}+Y_{i}^{L}}{2}$ is the center point of the interval, $Y_{i}^{W}=Y_{i}^{U}-Y_{i}^{C}$ is half the width of the interval and $\rho$ is the correlation matrix. An example of hybrid representation with random and multi-parallelepiped convex variables can be found in [39] where bounds on the probability of failure are estimated using Importance Sampling.

Evidence theory. Evidence theory (also called Dempster-Shafer (DS) theory) $[12,40]$ assigns weights to subsets $A$, also called "focal sets", of the power set $\Omega(Y)$ using the following mass distribution $\nu$ :

$$
\nu: \mid \begin{aligned}
\Omega(Y) & \longrightarrow[0,1] \\
A & \longrightarrow \nu(A) \text { s.t. } \sum_{A \in \Omega(Y)} \nu(A)=1 .
\end{aligned}
$$

Two measures can then be defined, namely the belief function $\operatorname{Bel}(\cdot)$ and the plausibility function $P l(\cdot)$, that bound the realization of any event $E$ :

$$
\begin{aligned}
& \operatorname{Bel}(E)=\sum_{A \subseteq E} \nu(A) \\
& \operatorname{Pl}(E)=\sum_{A \cap E \neq \emptyset} \nu(A) .
\end{aligned}
$$

The belief measure can be seen as an upper probability of the event $E$ while the plausibility measure can be seen as a lower probability. When combining evidence theory to a reliability analysis [41], the belief and plausibility 
measures enable to bound the probability of failure by considering the event $E=\{Y \in \mathcal{F}\}$. When the focal sets are singletons, the belief measure is equal to the plausibility measure and evidence theory reduces to probability theory. When there is only one focal set, it reduces to the interval representation.

Possibility theory. Possibility theory is a special case of evidence theory when focal sets are nested. Moreover, it is defined with the following possibility distribution $\pi$ :

$$
\pi: \Omega(Y) \rightarrow[0,1] \text { s.t. } \sup _{y \in \Omega(Y)} \pi(y)=1 .
$$

The triangular and trapezoidal distributions are common examples of possibility distributions. It follows the definition of two measures, namely the possibility $\Pi(\cdot)$ and the necessity $N(\cdot)$ :

$$
\begin{aligned}
& \Pi(E)=\sup _{y \in A} \pi(y) \\
& N(E)=\inf _{y \notin A}(1-\pi(y))
\end{aligned}
$$

where $E$ is any event. $\alpha$-cuts are commonly associated to a possibility distribution as they may be seen as nested confidence intervals with the following expression:

$$
\left[\underline{y}_{\alpha}, \bar{y}_{\alpha}\right]=\{y, \pi(y) \geq \alpha\} .
$$

Baudrit and Dubois [18] propose a method to jointly propagate probabilistic and possibilistic information. In [42], possibility distributions are assigned to the parameters of probability distributions to create fuzzy random variables and estimate fuzzy failure probabilities.

Probability boxes. The probability box (p-box) framework assigns an imprecise cumulative distribution function (cdf) to the uncertain variable $Y$. The true, yet uncertain cdf, is bounded by an upper $\operatorname{cdf} \bar{F}_{Y}$ and a lower $\operatorname{cdf} \underline{F}_{Y}$ such that:

$$
\bar{F}_{Y}(y) \leq F_{Y}(y) \leq \underline{F}_{Y}(y) .
$$

Two groups of p-boxes are distinguished, namely free p-boxes and parametric p-boxes. Free p-boxes do not make any further assumptions other than the bounds on the true cdf. Any shape that respects the bounds and the properties of a cdf is possible. Parametric p-boxes assume that the distribution 
type is known or, at the very least, belongs to a parametric family. The uncertainty lies in the parameters of the distribution (e.g., mean, variance) that are modeled using simple intervals. Therefore, for equal bounds, parametric p-boxes are more informative than free p-boxes by adding the information concerning the distribution type. A comparison between free and parametric p-boxes in the context of surrogate modeling for reliability assessment is proposed by Schöbi and Sudret [43]. Many uncertainty models already mentioned can be represented as free p-boxes. Indeed, by considering the event $\{Y \leq y\}$, plausibility and necessity measures can be seen as lower cdfs while belief and possibility measures can be seen as upper cdfs. Probability theory is retrieved when $\bar{F}_{Y}(y)=\underline{F}_{Y}(y)$. Monte Carlo sampling with p-box variables can be performed by using inverse sampling as shown in [44]. A review on more advanced techniques used to reduce the computational burden when propagating p-box variables is available in [45].

\section{References}

[1] M. Lemaire, Structural Reliability, Wiley \& Sons, 2009.

[2] E. Ardillon, et al., SRA into SRA: Structural reliability analyses into system risk assessment, an ESReDA collective book, Det Norske Veritas (2010).

[3] A. D. Kiureghian, Aleatory or epistemic? does it matter?, Structural Safety 31 (2009) 105-112.

[4] S. Göhler, T. Eifler, T. Howard, Robustness Metrics: Consolidating the multiple approaches to quantify Robustness, Journal of Mechanical Design 138 (2016) 111407.

[5] Y. Ben-Haïm, Info-Gap Decision Theory: Decisions under Severe Uncertainty, Elsevier, 2006.

[6] I. Takewaki, Y. Ben-Haïm, Info-gap robust design with load and model uncertainties, Journal of Sound and Vibrations 288 (2005) 551-570.

[7] Y. Kanno, S. Fujita, Y. Ben-Haïm, Structural design for earthquake resilience: Info-gap management uncertainty, Structural Safety 69 (2017) 23-33. 
[8] J. Hall, R. Lempert, K. Keller, A. Hackbarth, C. Mijere, D. McInerney, Robust climate policies under uncertainty: a comparison of robust decision making and info-gap methods, Risk Analysis 32 (2012) 1657-1672.

[9] E. Matrosov, A. Woods, J. Harou, Robust decision making and info-gap decision theory for water resource system planning, Journal of Hydrology 494 (2013) 43-58.

[10] A. Ajenjo, E. Ardillon, V.Chabridon, S. Cogan, E. Sadoulet-Reboul, Robustness evaluation of reliability assessments of penstocks using info-gap method, in: e-proceedings of the 30th European Safety and Reliability Conference and 15th Probabilistic Safety Assessment and Management Conference, Venice, Italy, 2020.

[11] Y. Ben-Haïm, I. Elishakoff, Convex models of uncertainty in applied mechanics, Elsevier, 1990.

[12] A. Dempster, Upper and lower probabilities induced by a multivalued mapping, The Annals of Mathematical Statistics 38 (1967) 325-339.

[13] D. Dubois, Possibility theory and statistical reasoning, Computational Statistics \& Data Analysis 51 (2006) 47-69.

[14] S. Ferson, L. Ginzburg, Different methods are needed to propagate ignorance and variability, Reliability Engineering \& System Safety 54 (1996) 133-144.

[15] M. Beer, S. Ferson, V. Kreinovich, Imprecise probabilities in engineering analyses, Mechanical Systems and Signal Processing 37 (2013) 4-29.

[16] E. Zio, N. Pedroni, Literature review of methods for representing uncertainty, Cahiers de la Sécurité Industrielle, Technical Report, Foundation for an Industrial Safety Culture (2013).

[17] F. Tonon, Using random set theory to propagate epistemic uncertainty through a mechanical system, Reliability Engineering \& System Safety 85 (2004) 169-181.

[18] C. Baudrit, D. Dubois, Joint propagation and exploitation of probabilistic and possibilistic information in risk assessment, IEEE Transactions on Fuzzy Systems 14 (2006) 593-608. 
[19] D. Alvarez, F. Uribe, J. Hurtado, Estimation of the lower and upper bounds on the probability of failure using subset simulation and random set theory, Mechanical Systems and Signal Processing 100 (2018) 782801.

[20] D. Alvarez, On the calculation of the bounds of probability of events using infinite random sets, International Journal of Approximate Reasoning 43 (2006) 241-267.

[21] D. Alvarez, J. Hurtado, J. Ramírez, Tighter bounds on the probability of failure than those provided by random set theory, Computers \& Structures 189 (2017) 101--113.

[22] D. Finkel, Direct optimization algorithm user guide, Technical Report, Center for Research in Scientific Computation, North Carolina State University (2003).

[23] Y. Ben-Haïm, Info-gap value of information in model updating, Mechanical Systems and Signal Processing 15 (2001) 457-474.

[24] R. Melchers, Importance sampling in structural systems, Structural Safety 6 (1989) 3-10.

[25] S. Au, J. Beck, Estimation of small failure probabilities in high dimensions by subset simulation., Probabilistic Engineering Mechanics 16 (2001) 263-277.

[26] J. Morio, M. Balesdent, Estimation of rare event probabilities in complex aerospace and other systems: a practical approach, Elsevier, 2015.

[27] A. Saltelli, S. Tarantola, F. Campolongo, M. Ratto., Sensitivity Analysis in Practice: A Guide to Assessing Scientific Models, Wiley, 2004.

[28] S. Ferson, W. Tucker, Sensitivity analysis using probability bounding, Reliability Engineering \& System Safety 91 (2006) 1435-1442.

[29] M. Baudin, A. Dutfoy, B. Iooss, A.-L. Popelin, OpenTURNS: An industrial software for uncertainty quantification in simulation, in: R. Ghanem, D. Higdon, H. Owhadi (Eds.), Handbook of Uncertainty Quantification, Springer, 2017, pp. 2001-2038. 
[30] A. D. Kiureghian, M. D. Stefano, Efficient algorithm for second-order reliability analysis, Journal of engineering mechanics 117 (1991) 29042923.

[31] V. Chabridon, M. Balesdent, J.-M. Bourinet, J. Morio, N. Gayton, Evaluation of failure probability under parameter epistemic uncertainty: application to aerospace system reliability assessment, Aerospace Science and Technology 69 (2017) 526-537.

[32] W. Dong, H. Shah, Vertex method for computing functions of fuzzy variables, Fuzzy Sets and Systems 24 (1987) 65-78.

[33] E. Ardillon, P. Bryla, A. Dumas, A semi-probabilistic approach for optimizing quantiles in the diagnoses of hydropower penstock pipes, Proceedings of the 54th ESReDA seminar (2018).

[34] P. Bryla, E. Ardillon, A. Dumas, Probabilistic models for penstock integrity assessment, Proceedings of the ESReL 2020 conference (2020).

[35] B. 7910, Guide to methods for assessing the acceptability of flaws in metallic structures., British Standard Institute (2015).

[36] X. Du, A. Sudjianto, B. Huang, Reliability-based design under the mixture of random and interval variables, Journal of Mechnical Design 127 (2005) 1068-1076.

[37] C. Jiang, G. Lu, L. Liu, A new reliability analysis method for uncertain structures with random and interval variables, International Journal of Mechanics and Materials in Design 8 (2012) 169-182.

[38] B. Ni, C. Jiang, X. Han, An improved multidimensional parallelepiped non-probabilistic model for structural uncertainty analysis, Applied Mathematical Modelling 40 (2016) 4727-4745.

[39] X. Liu, I. Elishakoff, A combined Importance Sampling and active learning Kriging reliability method for small failure probability with random and correlated interval variables., Structural Safety 82 (2020) 101875.

[40] G. Shafer, A mathematical theory of evidence, NJ: Princeton (1976). 
[41] Z. Zhang, C. Jiang, X. Han, D. Hu, S. Yu, A response surface approach for structural reliability analysis using evidence theory, Advances in Engineering Software 69 (2014) 37-45.

[42] M. Valdebenito, M. Beer, H. Jensen, J. Chen, P. Wei, Fuzzy failure probability estimation applying intervening variables, Structural Safety 83 (2020) 101909.

[43] R. Schöbi, B. Sudret, Structural reliability analysis for p-boxes using multi-level meta-models, Probabilistic Engineering Mechanics 48 (2017) $27-38$.

[44] H. Zhang, R. Mullen, R. Muhanna, Interval Monte Carlo methods for structural reliability, Structural Safety 32 (2010) 183-190.

[45] M. Faes, M. Daub, S. Marelli, E. Patelli, M. Beer, Engineering analysis with probability boxes: A review on computational methods., Structural Safety 93 (2021) 102092. 